\title{
Damage Identification of Beam Structures Using Free Response Shapes Obtained by Use of a Continuously Scanning Laser Doppler Vibrometer System
}

\author{
Y.F. Xu, Da-Ming Chen, W.D. Zhu* \\ Department of Mechanical Engineering, University of Maryland, Baltimore County, 1000 \\ Hilltop Circle, Baltimore, MD 21250, USA
}

\begin{abstract}
Spatially dense operating deflection shapes and mode shapes can be rapidly obtained by use of a continuously scanning laser Doppler vibrometer (CSLDV) system, which sweeps its laser spot over a vibrating structure surface. This paper introduces a new type of vibration shapes called a free response shape (FRS) that can be obtained by use of a CSLDV system, and a new damage identification methodology using FRSs is developed for beam structures. An analytical expression of FRSs of a damped beam structure is derived, and FRSs from the analytical expression compare well with those from a finite element model. In the damage identification methodology, a free-response damage index (FRDI) is proposed, and damage regions can be identified near neighborhoods with consistently high values of FRDIs associated with different modes; an auxiliary FRDI is defined to assist identification of the neighborhoods. A FRDI associated with a mode consists of differences be-
\end{abstract}

\footnotetext{
*Corresponding author. Tel: +1 410455 3394; fax: +1 4104551052.

Email addresses: yxu2@umbc.edu (Y.F. Xu), damingc1@umbc.edu (Da-Ming Chen), wzhu@umbc.edu (W.D. Zhu)
} 
tween curvatures of FRSs associated with the mode in a number of half-scan periods and those from polynomials that fit the FRSs with properly determined orders. A convergence index is proposed to determine the proper order of a polynomial fit. One advantage of the methodology is that the FRDI does not require any baseline information of an undamaged beam structure, if it is geometrically smooth and made of materials that have no stiffness and mass discontinuities. Another advantage is that FRDIs associated with multiple modes can be obtained using free response of a beam structure measured by a CSLDV system in one scan. The number of half-scan periods for calculation of the FRDI associated with a mode can be determined by use of the short-time Fourier transform. The proposed methodology was numerically and experimentally applied to identify damage in beam structures; effects of the scan frequency of a CSLDV system on qualities of obtained FRSs were experimentally investigated.

Keywords: damage identification; beam structure; continuously scanning laser Doppler vibrometer system; free response shape; free-response damage index

\section{Introduction}

Vibration-based damage detection has become a major research topic of structural dynamics in the past few decades [1]. Changes of physical properties of a structure, such as mass, stiffness and damping, are directly related to those of modal properties of the structure, i.e., natural frequencies, mode shapes (MSs) and modal damping ratios [2]. Damage that exist in a structure can be detected, located and characterized by use of modal character- 
istics. Methods that use changes of natural frequencies due to damage have been investigated by many researchers. They require a minimum amount of vibration measurement and can accurately detect damage, since natural frequencies are global characteristics of a structure and relatively easy to measure $[3,4,5,6,7,8]$. However, spatial information of structural property changes due to occurrence of damage cannot be directly obtained by use of natural frequencies, and one needs to construct accurate, physics-based models in order to apply the methods $[3,4,5,6,7,8]$, which can be difficult to achieve in practice, especially for complex large-scale structures. Since occurrence of damage can introduce local abnormalities in MSs near damage regions [9], unlike use of natural frequencies, the damage can be identified by inspecting smoothness of MSs without necessity of constructing models of structures. Being more sensitive to damage of small extent than MSs, curvatures of MSs (CMSs) are more often used to locate damage [10]. Effects of damage in a beam structure can be observed as severer local abnormalities in its CMSs than in MSs, and one can isolate the effects by comparing a CMS of the damaged beam structure with that of an undamaged one. It was shown that relatively large differences between a CMS of a damaged beam structure and that of an undamaged one mainly occur near a region of damage and the differences increase as severity of damage increases [10]. A gapped-smoothing method was proposed in Refs. $[11,12]$ to locate damage in beam structures by use of CMSs and curvatures of operating deflection shapes (ODSs), where those of undamaged beam structures are not needed. The gapped-smoothing method and a global fitting method were then synthesized to locate damage in beam structures [13, 14], where measured MSs 
and ODSs of damaged beam structures were fitted by generic MSs to approximate MSs of undamaged ones, and the method was further extended for plate structures [15]. CMS-based and wavelet-transform-based methods were proposed in Ref. [16] to identify embedded horizontal cracks in beam structures, where global trends of CMSs and wavelet transforms were eliminated by use MSs from polynomials that fit MSs of cracked beam structures with properly determined orders in a global manner.

A laser Doppler vibrometer (LDV) system is capable of accurate, noncontact surface vibration measurement; its mechanism is based on Doppler shifts between the incident light from and scattered light to the system [17]. An advantage of the system is that unwanted local stiffening and mass loading effects can be avoided, while use of attached transducers inevitably incurs such effects that can deteriorate accuracy of measurement, especially for lightweight structures. When equipped with a scanner that consists of a pair of orthogonal scan mirrors, a LDV becomes a scanning LDV. The laser beam from a scanning LDV can be directed to any visible position on a structure, and LDV measurement in an automatic manner can be achieved with a properly designed control scheme. However, it can take a relatively long acquisition time for a scanning LDV system to measure a large-scale structure with a dense measurement grid. The concept of a continuously scanning LDV (CSLDV) system was first proposed in Refs. [18, 19]. A CSLDV system continuously sweeps its laser over a surface of a structure under sinusoidal excitation to obtain its ODSs, which can be approximated by Chebysev series with coefficients determined by processing velocities measured by the system. Two CSLDV measurement methods were later proposed to obtain 
ODSs of a structure under sinusoidal excitation: demodulation and polynomial methods [20, 21]. In the demodulation method, velocities measured by a CSLDV system is modulated by multiplying a sinusoid at an excitation frequency, and a low-pass filter is applied to the modulated signal to obtain an ODS associated with the excitation frequency. In the polynomial method, it is assumed that an ODS can be represented by a polynomial, and coefficients of the polynomial can be determined by use of discrete Fourier transforms of measured velocities by a CSLDV system. The two methods were extended for structures under impact and multi-sine excitation in Refs. [22] and [23], respectively. A "lifting" method was proposed to obtain MSs from measured free response of a structure, where measured velocities by a CSLDV system are treated as output of linear time-periodic systems [24]. The "lifting" method was extended to output-only modal analysis to identify modal characteristics of a structure with use of harmonic transfer functions [25]. Use of a CSLDV system for damage identification was first proposed in Ref. [26], where the demodulation method was used to obtain ODSs of various structures by scanning their cracked surfaces, and effects of cracks could be observed as local abnormalities in obtained ODSs. The demodulation and polynomial methods were synthesized to identify damage in beams, where damage can be identified by use of a CSLDV system that scans intact surfaces of damaged beam structures [27].

In this work, a new type of vibration shapes called a free response shape (FRS) that can be obtained by use of a CSLDV system is introduced. An analytical expression of FRSs of a damped beam structure is derived. It is shown in the analytical expression that amplitudes of FRSs exponentially 
decay to zero with time. Numbers of non-zero FRSs associated with a mode can be determined by use of the short-time Fourier transform (STFT) of free response of the structure measured by a CSLDV system. A finite element model of a damped beam structure is constructed, and a CSLDV system is simulated to measure its free response. FRSs associated with the structure are obtained from the response maesured by the simulated CSLDV system from the demodulation method, and they are compared with those from the analytical expression. A new damage identification methodology that uses FRSs is proposed for beam structures. A free-response damage index (FRDI) is defined, which consists of differences between curvatures of FRSs obtained by use of a CSLDV system and those from polynomials that fit the FRSs, and damage regions can be identified near neighborhoods with consistently high values of FRDIs associated with different modes; an auxiliary FRDI is proposed to assist identification of the neighborhoods. A criterion based on a convergence index is proposed to determine orders of the polynomial fits. Effectiveness of the methodology for identifying damage in beam structures is numerically and experimentally investigated, and effects of the scan frequency of a CSLDV system on qualities of obtained FRSs were experimentally investigated.

\section{Methodology}

\subsection{Free response of a damped beam structure}

A linear time-invariant Euler-Bernoulli beam structure with a uniform cross-section is considered. The structure has a length $L$, a bending stiffness $E I$ and a linear mass density $m$. It is viscously damped, and damping 
effects are modeled using the Kelvin-Voigt viscoelastic model with a damping coefficient $c[28,29]$. Excitation in the form of a single impulse with an intensity $G_{0}$ is applied to the structure at position $x=L_{0}$ at time $t=0$. Response of the structure can be obtained by solving its governing partial differential equation

$$
E I\left[\frac{\partial^{4} y(x, t)}{\partial x^{4}}+c \frac{\partial}{\partial t}\left(\frac{\partial^{4} y(x, t)}{\partial x^{4}}\right)\right]+m \frac{\partial^{2} y(x, t)}{\partial t^{2}}=G_{0} \delta\left(x-L_{0}\right) \delta(t)
$$

with given boundary and initial conditions, where $y(x, t)$ is the displacement of the structure at position $x$ at time $t$. Based on the expansion theorem [28], a solution to Eq. (1) can be expressed by

$$
y(x, t)=\sum_{h=1}^{\infty} Y_{h}(x) F_{h}(t)
$$

where $Y_{h}(x)$ is the eigenfunction of the $h$-th mode of the corresponding undamped structure and $\eta_{h}(t)$ is the corresponding time function. The eigenfunction $Y_{h}(x)$ can be expressed by

$$
Y_{h}(x)=C_{1} \sin \beta_{h} x+C_{2} \cos \beta_{h} x+C_{3} \sinh \beta_{h} x+C_{4} \cosh \beta_{h} x
$$

where $C_{1}, C_{2}, C_{3}, C_{4}$ and $\beta_{h}$ are determined by the boundary conditions and the orthonormality condition of eigenfunctions

$$
\int_{0}^{L} m Y_{h}(x) Y_{j}(x) \mathrm{d} x=\delta_{h, j}
$$

in which $\delta_{h, j}$ is the Kronecker delta. The time function $F_{h}(t)$ can be obtained by solving an ordinary differential equation 


$$
\ddot{F}_{h}(t)+c\left(2 \pi f_{h}\right)^{2} \dot{F}_{h}(t)+\left(2 \pi f_{h}\right)^{2} F_{h}(t)=G_{0} Y_{h}\left(L_{0}\right) \delta(t)
$$

with initial conditions $F_{h}(0)$ and $\dot{F}_{h}(0)$ determined by those of Eq. (1), where $f_{h}$ is the natural frequency of the undamped structure in $\mathrm{Hz}$ associated with its $h$-th mode. A relation between $\beta_{h}$ and $f_{h}$ can be expressed by

$$
\beta_{h}^{4}=\frac{\left(2 \pi f_{h}\right)^{2} m}{E I}
$$

The solution to Eq. (5) can be expressed by [30]

$$
\begin{aligned}
F_{h}(t) & =\mathrm{e}^{-2 \pi \zeta_{h} f_{h} t}\left[F_{h}(0) \cos \left(2 \pi f_{h, d} t\right)+\left(\frac{\dot{F}_{h}(0)+2 \pi \zeta_{h} f_{h} F(0)}{2 \pi f_{h, d}}+\frac{G_{0} Y_{h}\left(L_{0}\right)}{2 \pi f_{h, d}}\right) \sin \left(2 \pi f_{h, d} t\right)\right] \\
& =A_{h} \mathrm{e}^{-2 \pi \zeta_{h} f_{h} t} \cos \left(2 \pi f_{h, d} t-\gamma_{h}\right)
\end{aligned}
$$

where

$$
A_{h}=\sqrt{\left(F_{h}(0)\right)^{2}+\left[\frac{\dot{F}_{h}(0)+2 \pi \zeta_{h} f_{h} F(0)}{2 \pi f_{h, d}}+\frac{G_{0} Y_{h}\left(L_{0}\right)}{2 \pi f_{h, d}}\right]^{2}}
$$

is an amplitude constant and

$$
\gamma_{h}=\arctan 2\left(\frac{\dot{F}_{h}(0)+2 \pi \zeta_{h} f_{h} F(0)}{2 \pi f_{h, d}}+\frac{G_{0} Y_{h}\left(L_{0}\right)}{2 \pi f_{h, d}}, F_{h}(0)\right)
$$

is a phase angle;

$$
\zeta_{h}=c \pi f_{h}
$$

and

$$
f_{h, d}=f_{h} \sqrt{1-\zeta_{h}^{2}}
$$


are the damping ratio and damped natural frequency of the structure associated with its $h$-th mode, respectively. Based on Eqs. (2) and $(7), y(x, t)$ can be expressed by

$$
y(x, t)=\sum_{h=1}^{\infty} A_{h} Y_{h}(x) \mathrm{e}^{-2 \pi \zeta_{h} f_{h} t} \cos \left(2 \pi f_{h, d} t-\gamma_{h}\right)
$$

\section{2. $F R S$}

A FRS associated with the $h$-th mode of the beam structure can be defined by

$$
\phi_{h}(x, t)=A_{h} Y_{h}(x) \mathrm{e}^{-2 \pi \zeta_{h} f_{h} t}
$$

and Eq. (12) becomes

$$
y(x, t)=\sum_{h=1}^{\infty} \phi_{h}(x, t) \cos \left(2 \pi f_{h, d} t-\gamma_{h}\right)
$$

It can be seen that $Y_{h}$, which can be considered as a MS associated with the $h$ th mode, exists in the definition of a FRS in Eq. (13). A similarity between $Y_{h}$ and $\phi_{h}$ is that they both correspond to the same mode of the structure. Since a MS describes amplitude ratios of displacement, velocity or acceleration at different positions on the structure while it vibrates, the multiplication factor of the MS can be an arbitrarily chosen non-zero constant, and the MS can be considered time-invariant. However, $\phi_{h}$ differs from $Y_{h}$ due to two extra terms in Eq. (13), i.e., $A_{h}$ and $\mathrm{e}^{-2 \pi f_{h} \xi_{h} t}$. The coefficient $A_{h}$ is determined by

the initial conditions of and impulse to the structure, and $\mathrm{e}^{-2 \pi f_{h} \xi_{h} t}$ indicates that amplitudes of $\phi_{h}$ at different positions exponentially decay to zero with time. Hence, $A_{h}$ cannot be arbitrarily chosen, and $\phi_{h}$ is time-varying. 
A CSLDV system continuously sweeps its laser spot over a vibrating structure surface with a specific scan pattern. It can measure response of a measurement point, where its laser spot is located during a scan, and a finite number of modes of a structure are included in free response measured by a CSLDV system. Let $\tilde{x}(t)$ be the position of a laser spot on a beam structure at time $t$; free response of the strucure measured by the CSLDV system with a straight scan line along its length can be expressed by

$$
\tilde{y}(t)=\sum_{h=1}^{n} \tilde{\phi}_{h}(\tilde{x}(t)) \tilde{\eta}_{h}(t)
$$

where $n$ is the number of measured modes, and $\tilde{\phi}_{h}$ and $\tilde{\eta}_{h}$ are the FRS and time function associated with the $h$-th mode measured by the system, respectively. The FRS $\tilde{\phi}_{h}$ in Eq. (15) can be defined in a way similar to $\phi_{h}$ in Eq. (13):

$$
\tilde{\phi}_{h}(t)=A_{h} Y_{h}(\tilde{x}(t)) \mathrm{e}^{-2 \pi f_{h} \zeta_{h} t}
$$

A major difference between $\phi_{h}$ in Eq. (13) and $\tilde{\phi}_{h}$ is that $x$ in the former becomes $\tilde{x}$ in the latter, which is a function of $t$ and is unique in a scan of the system. Similar to $\phi_{h}, \tilde{\phi}_{h}$ contains both $A_{h}$ and $\mathrm{e}^{-2 \pi f_{h} \zeta_{h} t}$, and it is time-varying. The time function $\tilde{\eta}_{h}$ can be expressed by

$$
\tilde{\eta}_{h}(t)=\cos \left(2 \pi f_{h} t-\alpha_{h}-\theta_{h}\right)
$$

where $\alpha_{h}$ is the difference between a phase determined by the initial conditions and impulse associated with the $h$-th mode and that by a mirror feedback signal, and $\theta_{h}$ is a phase variable that controls amplitudes of in- 
phase and quadrature components of $\tilde{\phi}_{h}$, which can be expressed by

$$
\tilde{\phi}_{I, h}=\tilde{\phi}_{h} \cos \left(\alpha_{h}+\theta_{h}\right)
$$

and

$$
\tilde{\phi}_{Q, h}=\tilde{\phi}_{h} \sin \left(\alpha_{h}+\theta_{h}\right)
$$

respectively [27].

\subsection{Demodulation method for FRSs}

The demodulation method has been proposed to obtain ODSs of a structure under sinusoidal excitation [21], where its steady-state response measured by a CSLDV system are analyzed. FRSs of a linear damped beam structure measured by a CSLDV system, as described by $\tilde{\phi}_{h}$ in Eq. (16), can also be obtained from the demodulation method by analyzing its free response of half-scan periods measured by the system, and each obtained $\tilde{\phi}_{h}$ corresponds to a mode in a half-scan period. A half-scan period starts when the laser spot of the system arrives at one end of a scan line, and it ends when the laser spot arrives at the other end. Hence, multiple $\tilde{\phi}_{h}$ can be obtained from free response of the structure measured by the system in one scan. To identify the start and end of a half-scan period, one can refer to mirror feedback signals of a CSLDV system and determine instants when its laser spot arrives at ends of a scan line.

Application of the demodulation method for obtaining $\tilde{\phi}_{h}$ associated with the $h$-th mode in a half-scan period measured by a CSLDV system is described below. Based on Eqs. (15) through (19), a half-scan period of free 
response of the structure that is measured by a CSLDV system can be expressed by

$$
\begin{aligned}
\tilde{y}(t) & =\sum_{h=1}^{n} \tilde{\phi}_{h}(\tilde{x}(t)) \cos \left(2 \pi f_{h} t-\alpha_{h}-\theta_{h}\right) \\
& =\sum_{h=1}^{n}\left[\tilde{\phi}_{I, h}(\tilde{x}(t)) \cos \left(2 \pi f_{h} t\right)+\tilde{\phi}_{Q, h}(\tilde{x}(t))(x, t) \sin \left(2 \pi f_{h} t\right)\right]
\end{aligned}
$$

The response $\tilde{y}(t)$ is then multiplied by $\cos \left(2 \pi f_{k} t\right)$ and $\sin \left(2 \pi f_{k} t\right)$, which gives

$$
\begin{aligned}
\tilde{y}(t) \cos \left(2 \pi f_{k} t\right)= & \tilde{\phi}_{I, k}(\tilde{x}(t)) \cos \left(2 \pi f_{k} t\right) \cos \left(2 \pi f_{k} t\right)+\tilde{\phi}_{Q, k}(\tilde{x}(t)) \sin \left(2 \pi f_{k} t\right) \cos \left(2 \pi f_{k} t\right)+ \\
& \sum_{h=1, h \neq k}^{n} \tilde{\phi}_{h}(\tilde{x}(t)) \tilde{\eta}_{h}(t) \cos \left(2 \pi f_{k} t\right) \\
= & \frac{1}{2} \tilde{\phi}_{I, k}(\tilde{x}(t))+\frac{1}{2} \tilde{\phi}_{I, k}(\tilde{x}(t)) \cos \left(4 \pi f_{k} t\right)+\frac{1}{2} \tilde{\phi}_{Q, k}(\tilde{x}(t)) \sin \left(4 \pi f_{k} t\right)+ \\
& \sum_{h=1, h \neq k}^{n} \tilde{\phi}_{h}(\tilde{x}(t)) \tilde{\eta}_{h}(t) \cos \left(2 \pi f_{k} t\right)
\end{aligned}
$$

and

$$
\begin{aligned}
\tilde{y}(t) \sin \left(2 \pi f_{k} t\right)= & \tilde{\phi}_{Q, k}(\tilde{x}(t)) \cos \left(2 \pi f_{k} t\right) \sin \left(2 \pi f_{k} t\right)+\tilde{\phi}_{Q, k}(\tilde{x}(t)) \sin \left(2 \pi f_{k} t\right) \sin \left(2 \pi f_{k} t\right)+ \\
& \sum_{h=1, h \neq k}^{n} \tilde{\phi}_{h}(\tilde{x}(t)) \tilde{\eta}_{h}(t) \sin \left(2 \pi f_{k} t\right) \\
= & \frac{1}{2} \tilde{\phi}_{Q, k}(\tilde{x}(t))-\frac{1}{2} \tilde{\phi}_{Q, k}(\tilde{x}(t)) \cos \left(4 \pi f_{k} t\right)+\frac{1}{2} \tilde{\phi}_{I, k}(\tilde{x}(t)) \sin \left(4 \pi f_{k} t\right)+ \\
& \sum_{h=1, h \neq k}^{n} \tilde{\phi}_{h}(\tilde{x}(t)) \tilde{\eta}_{h}(t) \sin \left(2 \pi f_{k} t\right)
\end{aligned}
$$

respectively. A low-pass filter is then applied to $\tilde{y}(t) \cos \left(2 \pi f_{k} t\right)$ and $\tilde{y}(t) \sin \left(2 \pi f_{k} t\right)$ in Eqs. (21) and (22) to obtain $\frac{1}{2} \tilde{\phi}_{I, k}$ and $\frac{1}{2} \tilde{\phi}_{Q, k}$, respectively, and the second and third terms on the third lines and terms on the fourth lines of Eqs. (21) 
and (22) are removed. Further, $\tilde{\phi}_{I, k}$ and $\tilde{\phi}_{Q, k}$ can be obtained by multiplying the corresponding filtered signals by two. The value of $\theta_{h}$ in Eq. (20) can be optimized so that $\tilde{\phi}_{I, h}$ and $\tilde{\phi}_{Q, h}$ attain their maximum and minimum amplitudes, respectively. In what follows, all FRSs are represented by their in-phase components with maximum amplitudes.

\subsection{FRDI}

It is shown in Sec. 2.2 that a FRS corresponds to a mode of a beam structure, and its amplitude is time-varying and exponentially decays to zero with time. In order to obtain non-zero FRSs from the demodulation method, one needs to determine natural frequencies of the structure and instants when amplitudes of the FRSs decay to zero. While the natural frequencies can be determined by use of the fast Fourier transform of $\tilde{y}(t)$, the instants can be determined by use of the STFT of $\tilde{y}(t)$ [31], denoted by $\tilde{V}_{w}(t, f)$, which can be expressed by

$$
\tilde{V}_{w}(t, f)=\int_{-\infty}^{\infty} \tilde{y}(\tau) g_{s}^{*}(\tau-t) \mathrm{e}^{-2 \pi \mathrm{j} f \tau} \mathrm{d} \tau
$$

where $g_{s}$ is a window function with a scale $s$, the superscript $*$ denotes complex conjugation, and $\mathrm{j}=\sqrt{-1}$. The scale $s$ determines the width of $g_{s}$ in the time domain, which should be smaller than that of a half-scan period. When $\tilde{V}_{w}$ at the natural frequency associated with the $h$-th mode becomes almost zero at an instant $t_{h, 0}$, the amplitude of $\tilde{\phi}_{h}$ is considered to be zero. Multiple non-zero $\tilde{\phi}_{h}$ can be obtained using $\tilde{V}_{w}$ of the first $N_{h}$ half-scan periods, where $N_{h}$ is an integer that can be defined as 


$$
\arg \max _{N_{h}} \frac{N_{h} T_{h}}{2} \leqslant t_{h, 0}-t_{1}
$$

in which $T$ is the length of a scan period and $t_{1}$ is the instant when the first half-scan period starts. In this work, $\tilde{V}_{w}(t, f)$ is visualized by use of a spectrogram whose intensity denotes the power spectral density associated with $\tilde{V}_{w}(t, f)$ in Eq. (23), where $g_{s}$ is a Hamming function that can be expressed by

$$
g_{s}(t)= \begin{cases}0.54-0.46 \cos \left(\frac{2 \pi t}{s}\right) & , 0 \leqslant t \leqslant s \\ 0 & , \text { otherwise }\end{cases}
$$

A CMS $Y_{h}^{\prime \prime}$ is the second-order spatial derivative of $Y_{h}$, where a prime denotes first-order spatial differentiation with respect to $x$. A curvature FRS $\tilde{\phi}_{h}^{\prime \prime}$ can be defined as

$$
\tilde{\phi}_{h}^{\prime \prime}(\tilde{x}(t))=\frac{\partial^{2} \tilde{\phi}_{h}}{\partial \tilde{x}^{2}}=A_{h} Y_{h}^{\prime \prime}(\tilde{x}(t)) \mathrm{e}^{-2 \pi f_{h} \zeta_{h} t}
$$

Since $Y_{h}^{\prime \prime}$ is related to bending stiffness of a beam structure that can decrease due to occurrence of damage and regions of the decrease correspond to damage regions, it can be used for damage identification [10], and so can $\tilde{\phi}_{h}^{\prime \prime}$, since it explicitly contains $Y_{h}^{\prime \prime}$ as shown in Eq. (26).

Since a MS of an undamaged beam structure can be well approximated by that from a polynomial that fits a MS of a damaged beam structure [16], it can be inferred that a FRS of an undamaged structure can also be well approximated by that from a polynomial that fits a FRS of a damaged structure. A damage index similar to that in Ref. [16] can be defined by comparing $\tilde{\phi}_{h}^{\prime \prime}$ of a damaged beam structure and that from a polynomial that 
fits $\tilde{\phi}_{h}$ with a properly determined order, which can be expressed by

$$
\delta_{h}(\tilde{x})=\sum_{i=1}^{N_{d}}\left[\tilde{\phi}_{h, i}^{\prime \prime}(\tilde{x})-\tilde{\phi}_{h, i}^{p \prime \prime}(\tilde{x})\right]^{2}
$$

where $N_{d}$ is the number of FRSs to be included in the index with $N_{d} \leq N_{h}$, $\tilde{\phi}_{h, i}$ is a FRS associated with the $h$-th mode in the $i$-th half-scan period, and $\tilde{\phi}_{h, i}^{p}$ is a FRS from a polynomial that fits $\tilde{\phi}_{h, i}$ with a properly determined order. The index $\delta_{h}(\tilde{x})$ in Eq. (27) is termed as a free-response damage index (FRDI) at $\tilde{x}$. Since there can be FRSs associated with multiple modes corresponding to $\tilde{y}$ measured by a CSLDV system in one scan, FRDIs associated with multiple modes can be obtained using measurement by a CSLDV system in one scan, and damage regions can be identified near neighborhoods with consistently high values of FRDIs associated with different modes. Note that use of $\delta_{h}$ associated with rigid-body modes should be excluded in damage identification as curvatures of their FRSs are zero, and one should use $\delta_{h}$ associated with elastic modes in damage identification. An auxiliary FRDI associated with the FRDI in Eq. (27) can be defined to assist identification of the neighborhoods, which can be expressed by

$$
\delta_{\mathrm{a}}(\tilde{x})=\sum_{h=1}^{H} \hat{\delta}_{h}(\tilde{x})
$$

where $H$ is the number of FRDIs to be included in the auxiliary index and $\hat{\delta}_{h}(\tilde{x})$ is a normalized FRDI associated with the $h$-th mode with the maximum amplitude of one. Since border distortions occur in curvature ODSs associated with ODSs obtained from the demodulation method [27], similar distortions would also occur in curvature FRSs associated with FRSs obtained from the demodulation method. Hence, regions containing such 
distortions are excluded in presenting $\delta_{h}(\tilde{x})$ and $\delta_{\mathrm{a}}(\tilde{x})$ and in normalization of $\delta_{h}(\tilde{x})$ in $\delta_{\mathrm{a}}(\tilde{x})$. Neighborhoods with consistently high values of the FRDIs associated with the $H$ modes can be identified in those with high values of the auxiliary FRDI.

A polynomial that fits $\tilde{\phi}_{h, i}$ with an order $r$ can be expressed by

$$
\tilde{\phi}_{h, i}^{p}(\tilde{x})=\sum_{q=0}^{r} a_{q} \tilde{x}^{q}
$$

where $a_{q}$ are coefficients of the polynomial that can be obtained by solving a linear equation

$$
\mathbf{U} \mathbf{a}=\tilde{\Phi}
$$

in which $\mathbf{U}$ is an $M \times(r+1)$ Vandermonde matrix with $M$ being the number of measurement points of $\tilde{\phi}_{h, i}$ :

$$
\mathbf{U}=\left[\begin{array}{ccccc}
1 & \tilde{x}_{1} & \tilde{x}_{1}^{2} & \cdots & \tilde{x}_{1}^{r} \\
1 & \tilde{x}_{2} & \tilde{x}_{2}^{2} & \cdots & \tilde{x}_{2}^{r} \\
\vdots & \vdots & \vdots & \ddots & \vdots \\
1 & \tilde{x}_{M} & \tilde{x}_{M}^{2} & \cdots & \tilde{x}_{M}^{r}
\end{array}\right]
$$

$\mathbf{a}=\left[\begin{array}{llll}a_{0} & a_{1} & \ldots & a_{r}\end{array}\right]^{\mathrm{T}}$ is an $(r+1)$-dimensional coefficient vector, and $\tilde{\boldsymbol{\Phi}}$ is the FRS vector of the structure to be fit. To avoid ill-conditioning of $\mathbf{U}$, it is proposed that $\tilde{x}$ in Eq. (29) be normalized using the "center and scale" technique [32] before formulation of the linear equation in Eq. (29). Normalized coordinates $\hat{x}$ can be expressed by

$$
\hat{x}=\frac{2 \tilde{x}-2 \bar{x}}{l}
$$


where $\bar{x}$ is the $x$-coordinate of the center point of a scan line and $l$ is its length.

As pointed out in Ref. [16], an increase of $r$ in the polynomial in Eq. (29) can improve the level of approximation of $\tilde{\phi}_{h, i}^{p}$ to $\tilde{\phi}_{h, i}$. To determine the proper order of the polynomial fit, a convergence index can be defined:

$$
\operatorname{con}(r)=\frac{\operatorname{RMS}(\tilde{\boldsymbol{\Phi}})}{\operatorname{RMS}(\tilde{\boldsymbol{\Phi}})+\operatorname{RMS}(\mathbf{e})} \times 100 \%
$$

where RMS (.) denotes the root-mean-square value of a vector and $\mathbf{e}=\mathbf{U a}-$

$\tilde{\boldsymbol{\Phi}}$ is an error vector. When the convergence index is close to $100 \%, \phi_{h, i}^{p}$ converges to $\phi_{h, i}$; the lower the index, the lower the level of convergence of $\phi_{h, i}^{p}$ to $\phi_{h, i}$. It is proposed in this work that the proper value of $r$ be two plus the least value of $r$ with which con $(r)$ is above 95\%. Two is added here in order to preserve smoothness of a curvature FRS from the polynomial fit, since calculation of a curvature incurs second-order differentiation that reduces the order of a polynomial by two.

\section{Numerical Investigation}

\subsection{FRSs from analytical and FE models}

Based on Eq. (1), the analytical model of an undamaged aluminum cantilever beam structure with $L=0.8 \mathrm{~m}, E=68.9 \mathrm{GPa}, m=0.2700 \mathrm{~kg} / \mathrm{m}$ and $c=8 \times 10^{-7} \mathrm{~s}$ is formulated; the structure has a uniform square crosssection with a side length of $0.01 \mathrm{~m}$. The structure has fixed and free ends at $x=0$ and $x=L$, respectively, and it has zero initial conditions. A single impulse with an intensity of $0.01 \mathrm{~N} \cdot \mathrm{s}$ is applied to the free end of the 
structure. A corresponding FE model of the structure under the same initial conditions and excitation is constructed using ABAQUS with 16384 two-node linear beam (B21) elements for comparison purposes, where the damping in the analytical model can be equivalently modeled using the Rayleigh damping [28]. The formulation of the FE model can be expressed by

$$
\mathbf{M} \ddot{\mathbf{z}}(t)+\mathbf{C} \dot{\mathbf{z}}(t)+\mathbf{K z}(t)=\mathbf{f}(t)
$$

with initial conditions $\mathbf{z}(0)=\mathbf{0}$ and $\dot{\mathbf{z}}(0)=\mathbf{0}$, where $\mathbf{M}, \mathbf{C}$ and $\mathbf{K}$ are mass, damping and stiffness matrices, respectively, in which $\mathbf{C}=\alpha \mathbf{M}+\beta \mathbf{K}$ with Rayleigh damping coefficients $\alpha=0$ and $\beta=c$, i.e., $\mathbf{C}=c \mathbf{K}$, and $\mathbf{z}$ and $\mathbf{f}$ are displacement and force vectors, respectively. Table 1 compares damped natural frequencies of the first five modes of the structure from its analytical and FE models, and the largest difference between the damped natural frequencies from the two models is $0.61 \%$. Note that differences between the natural frequencies of the two models arise due to use of the B21 elements and they can be greatly reduced if two-node cubic beam (B22) elements were used. Mass-normalized MSs of the first five modes from the analytical and FE models compare well, as shown in Fig. 1. 
Tab. 1 Comparison between damped natural frequencies of the cantilever beam structure from its analytical and FE models.

\begin{tabular}{|c|c|c|c|}
\hline Mode & Analytical Frequency $(\mathrm{Hz})$ & FE Frequency $(\mathrm{Hz})$ & Difference (\%) \\
\hline \hline 1 & 12.75 & 12.75 & 0.01 \\
\hline 2 & 79.91 & 79.84 & 0.09 \\
\hline 3 & 223.74 & 223.28 & 0.21 \\
\hline 4 & 438.44 & 436.77 & 0.38 \\
\hline 5 & 724.77 & 720.38 & 0.61 \\
\hline
\end{tabular}

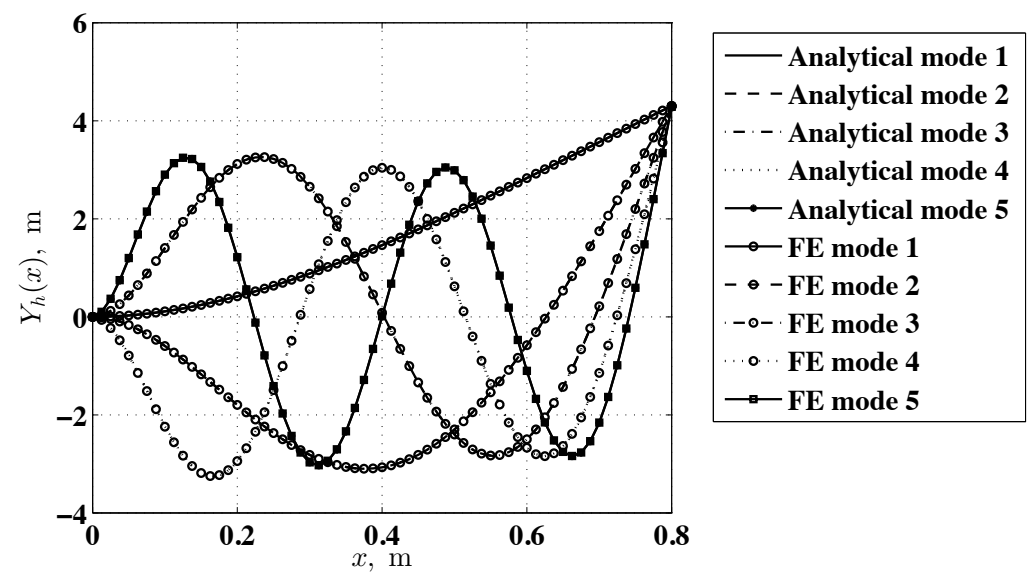

Fig. 1 Mass-normalized MSs of the cantilever beam structure associated with its first five modes from its analytical and FE models.

Response of the beam structure is then measured by a simulated CSLDV system with a scan period of $T=2 \mathrm{~s}$ and a sampling frequency of 16384 $\mathrm{Hz}$; the simulated CSLDV system is capable of measuring response in the 
form of displacement. The position of its laser spot is shown in Fig. 2(a); the first half-scan period starts at $t=0.0625 \mathrm{~s}$, and measured response of the structure from the analytical and FE models in the first half-scan period, second half-scan period and first eight seconds are shown in Figs. 2(b) through $(d)$, respectively. Spectrograms of the response from the analytical and FE models in the first eight seconds are shown in Figs. 3(a) and (b), respectively, and they compare well with each other. It can be seen from Figs. 3(a) and (b) that STFTs at the first and second natural frequencies of the structure do not decay much in the first eight seconds, while those at the third through fifth natural frequencies decay more. More importantly, the STFTs associated with the fifth natural frequency of the structure drastically decay within the first second of the CSLDV measurement. As a result, FRSs associated with the fifth elastic mode cannot be obtained in this scan. Hence, only FRSs associated with the first four elastic modes can be obtained from the response. 


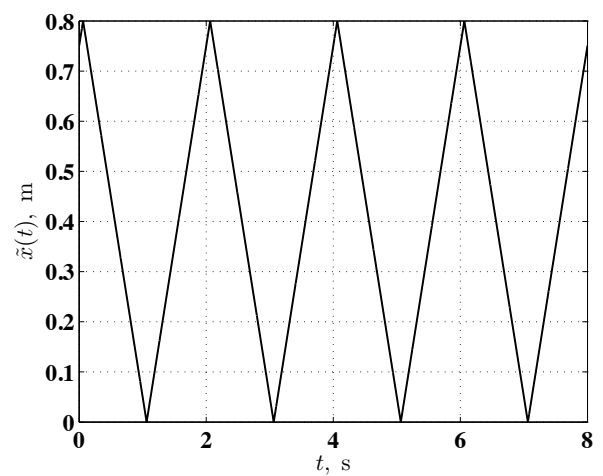

(a)

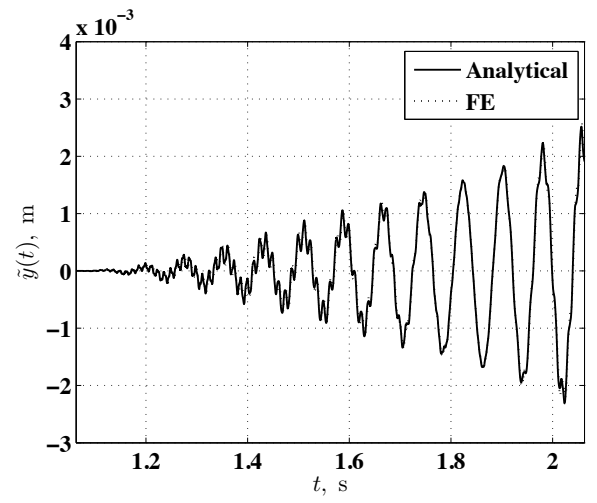

(c)

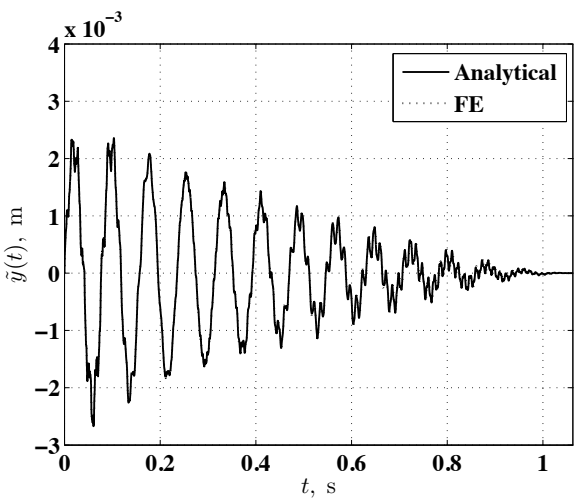

(b)

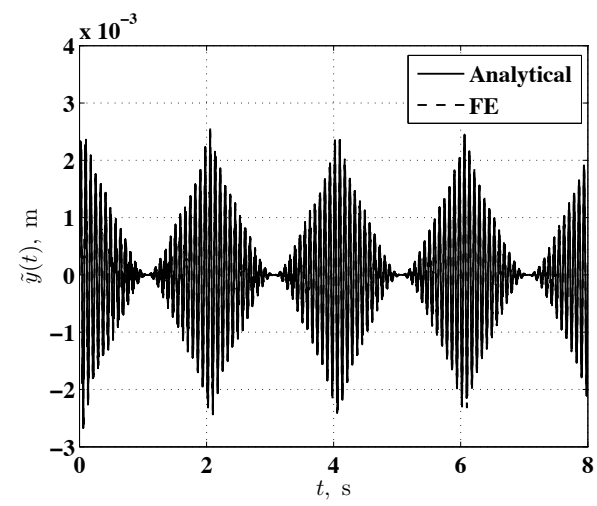

(d)

Fig. 2 (a) Position of the laser spot of a simulated CSLDV system on the beam structure, (b) response from its analytical and FE models measured by the simulated CSLDV system in the first half-scan period, (c) response from its analytical and FE models measured by the simulated CSLDV system in the second halfscan period and (d) response from its analytical and FE models measured by the simulated CSLDV system in the first eight second. 


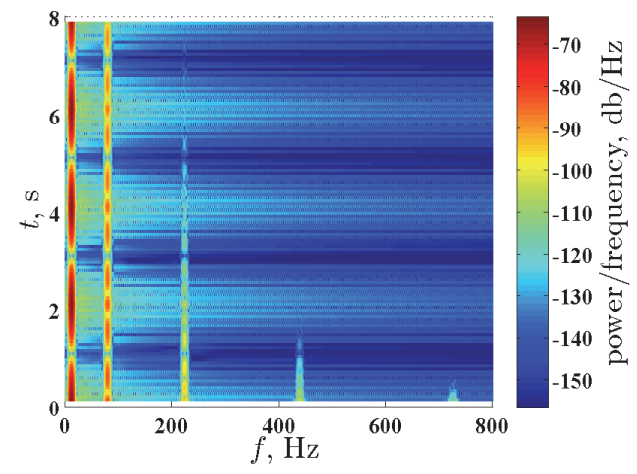

(a)

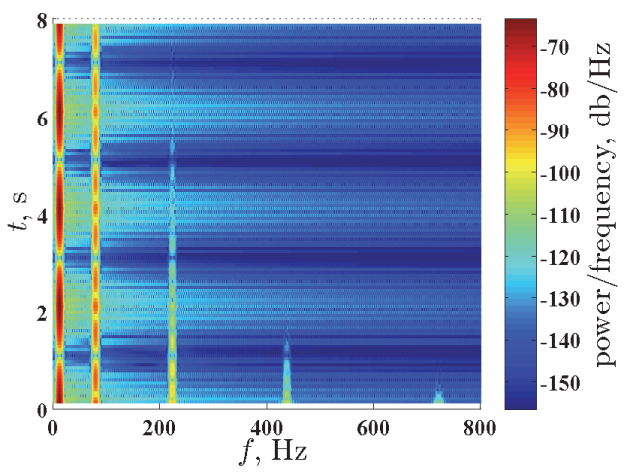

(b)

Fig. 3 Spectrograms of the response of the beam structure from its (a) analytical and (b) FE models measured by the simulated CSLDV system shown in Fig. 2(d).

Based on Eq. (16), FRSs from the analytical model associated with the first four modes of the beam structure in the first three half-scan periods of the simulated CSLDV system are shown in Figs. 4(a) through (d), respectively. FRSs from the FE model obtained by use of the simulated CSLDV system, which are obtained from the demodulation method, are shown in Fig. 5. It can be seen from Figs. 4 and 5 that the FRSs from the analytical and FE models are in good agreement. Amplitudes of the FRSs associated with the fourth mode of the structure in the second and third half-scan periods drastically decrease to almost zero due to the damping; the FRS in the first half-scan period is included in $\delta_{h}(\tilde{x})$ associated with the fourth mode for damage identification that follows. 


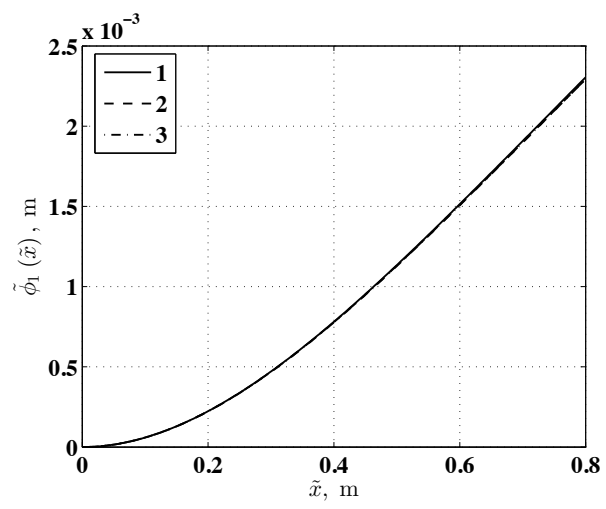

(a)

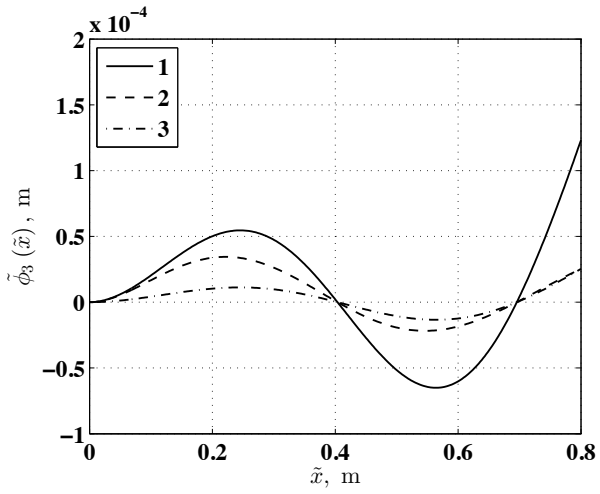

(c)

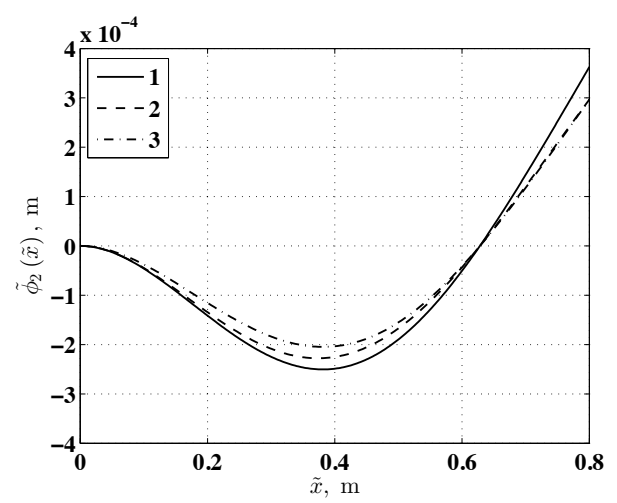

(b)

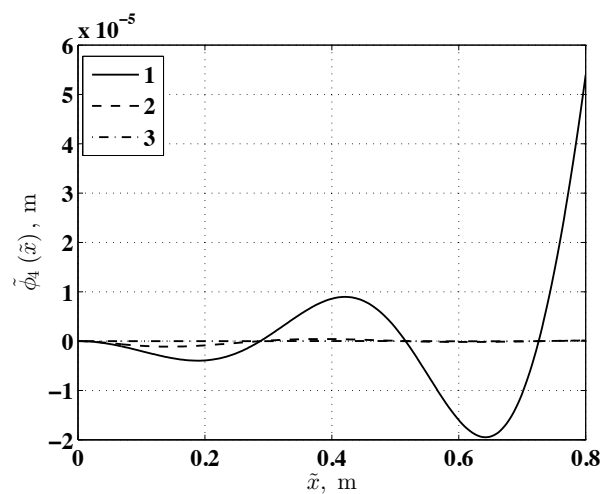

(d)

Fig. 4 FRSs of the beam structure from its analytical model associated with its (a) first, (b) second, (c) third and (d) fourth modes in the first three half-scan periods of the simulated CSLDV system. 


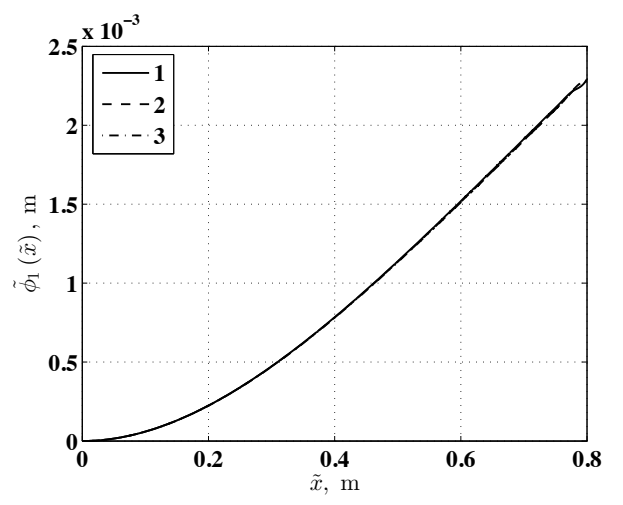

(a)

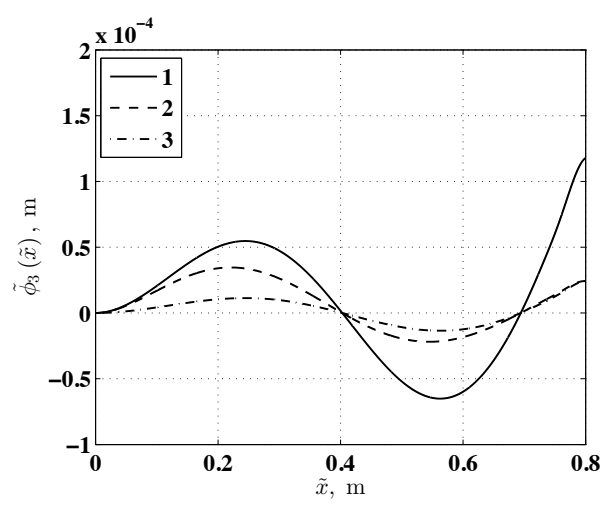

(c)

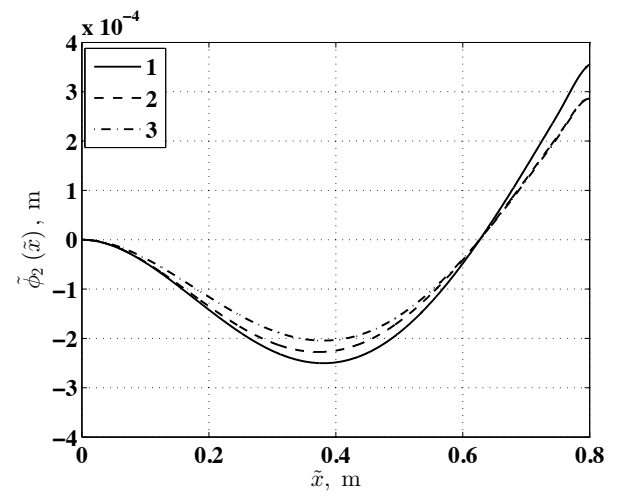

(b)

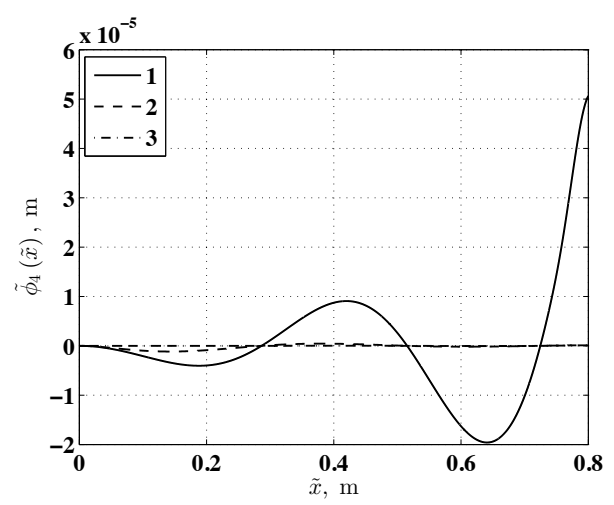

(d)

Fig. 5 FRSs of the beam structure from its FE model associated with its (a) first, (b) second, (c) third and (d) fourth modes obtained by use of the simulated CSLDV system in the first three half-scan periods.

\subsection{Damage identification using FRDIs}

Since fidelity of the FE model of the undamaged cantilever beam structure has been validated in Sec. 3.1, the FE model can be adapted to model such a beam structure with damage in the form of thickness reduction to numerically investigate the proposed damage identification methodology. The thickness of the section of the structure between $x=\frac{6}{16} L$ and $x=\frac{7}{16} L$ is 
reduced by $10 \%$, while its $E$ and volume mass density remain unchanged. Table 2 compares natural frequencies of the first five modes of the damaged and undamaged structures from the FE models, and the largest difference is $1.27 \%$. Response of the damaged structure from its FE model is measured by the simulated CSLDV system with the same settings as those in Sec. 3.1, and it is used to obtain FRSs of the damaged structure associated with the first four modes in the first three half-scan periods, as shown in Fig. 6.

Tab. 2 Comparison between damped natural frequencies of the damaged and undamaged cantilever beam structures from their FE models.

\begin{tabular}{|c|c|c|c|}
\hline Mode & Damaged Frequency $(\mathrm{Hz})$ & Undamaged Frequency $(\mathrm{Hz})$ & Difference $(\%)$ \\
\hline \hline 1 & 12.64 & 12.75 & -0.88 \\
\hline 2 & 79.04 & 79.84 & -1.00 \\
\hline 3 & 222.03 & 223.28 & -0.56 \\
\hline 4 & 434.80 & 436.77 & -0.45 \\
\hline 5 & 711.23 & 720.38 & -1.27 \\
\hline
\end{tabular}




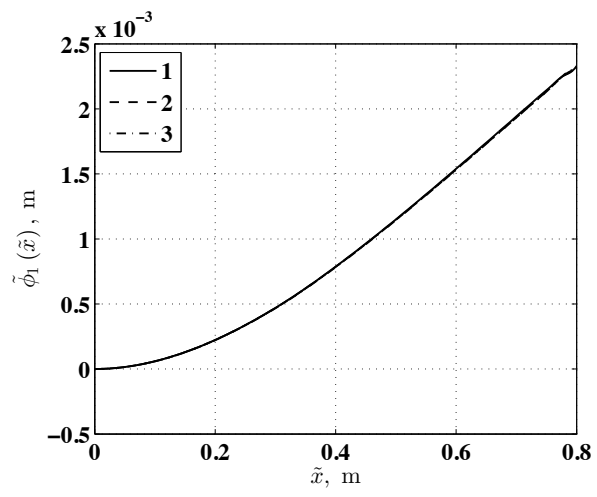

(a)

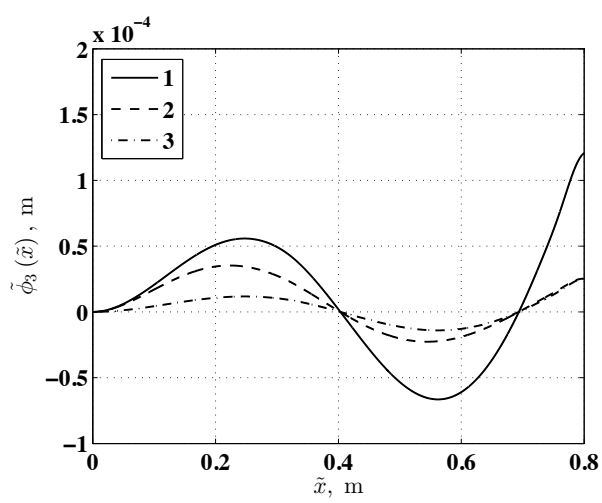

(c)

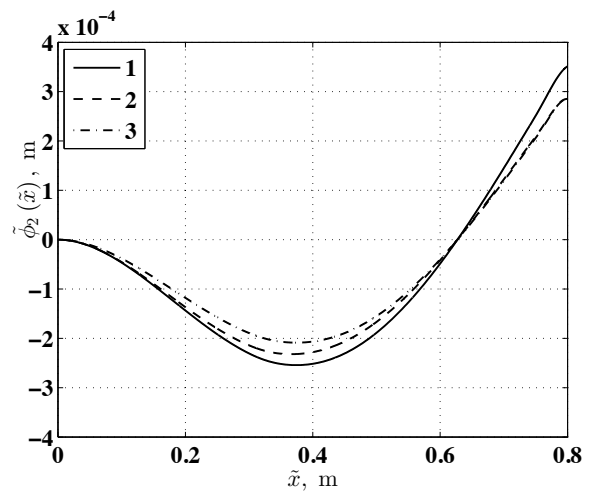

(b)

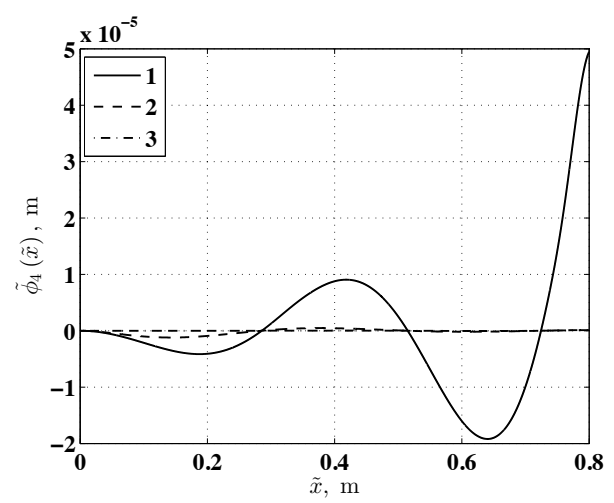

(d)

Fig. 6 FRSs from the FE model of the damaged beam structure obtained by use of the simulated CSLDV system associated with the (a) first, (b) second, (c) third and (d) fourth modes in the first three half-scan periods.

Convergence indices con corresponding to the FRSs associated with the first through fourth modes of the damaged beam structure are shown in Figs. 7 through 10, respectively. Proper orders of polynomials that fit the FRSs associated with the first mode in the first through third half-scan periods are determined to be 4, 4 and 4, respectively; those associated with the second mode are determined to be 6,6 and 6 , respectively; those associated with the 
third mode are determined to be 7, 7 and 7, respectively; and that associated with the fourth mode is determined to be 9 .

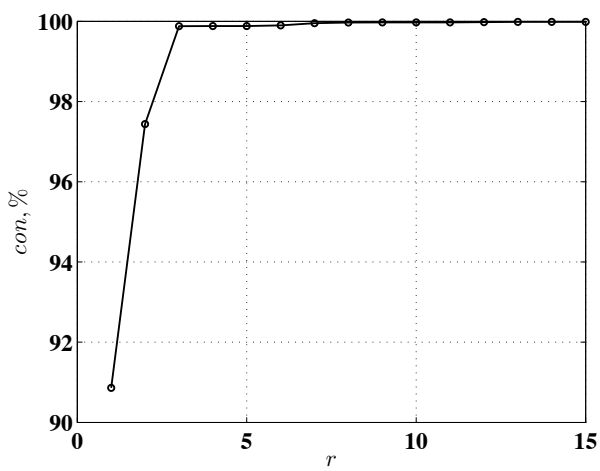

(a)

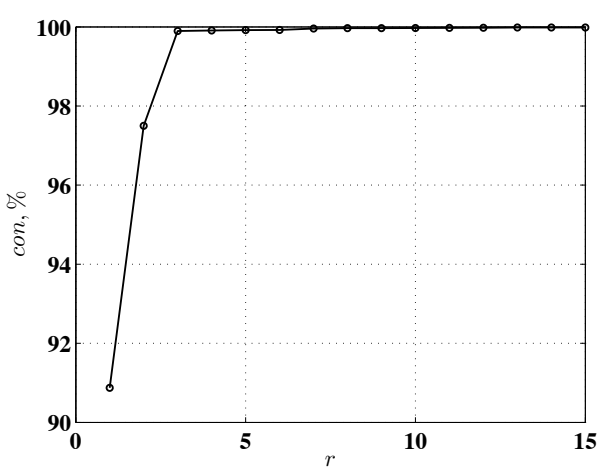

(b)

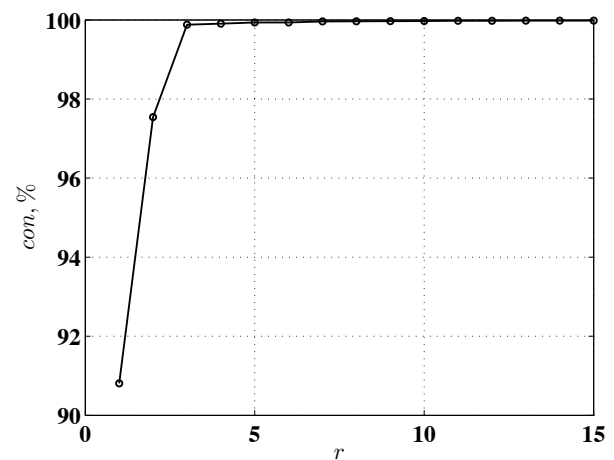

(c)

Fig. 7 Convergence indices con corresponding to the FRSs associated with the first mode in the (a) first, (b) second and (c) third half-scan periods. 


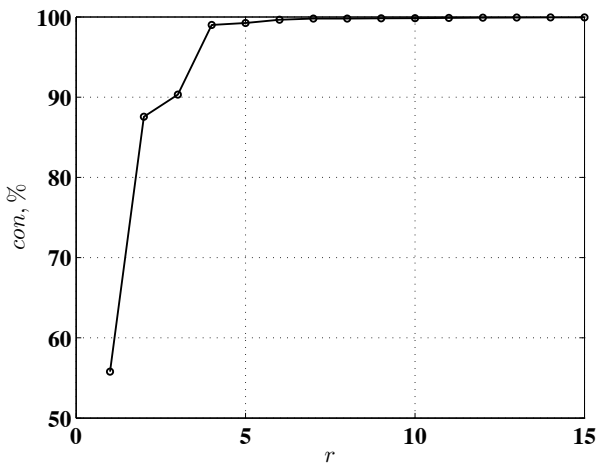

(a)

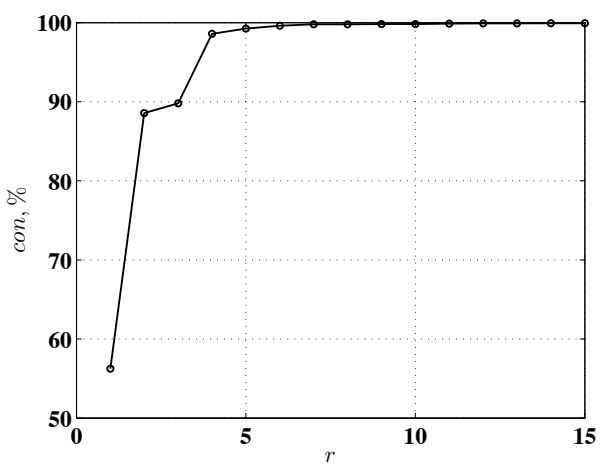

(b)

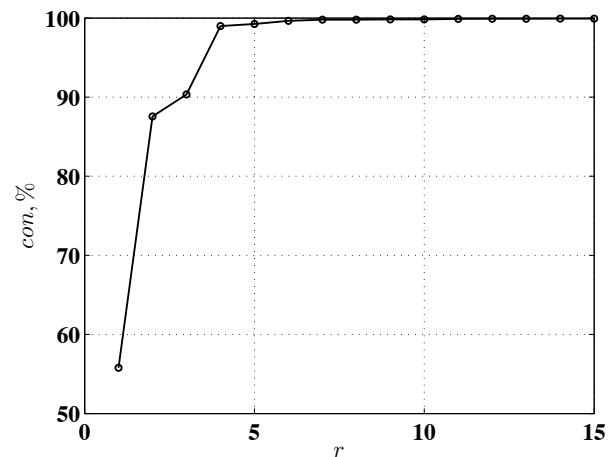

(c)

Fig. 8 Convergence indices con corresponding to the FRSs associated with the second mode in the (a) first, (b) second and (c) third half-scan periods. 


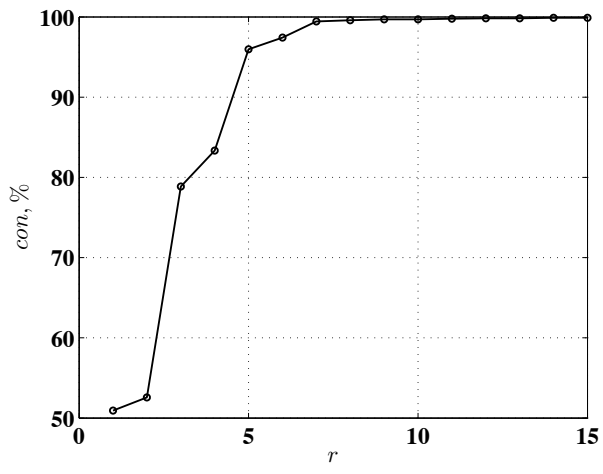

(a)

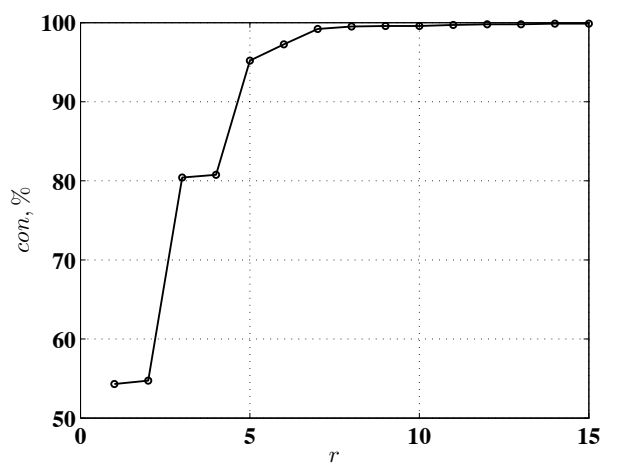

(b)

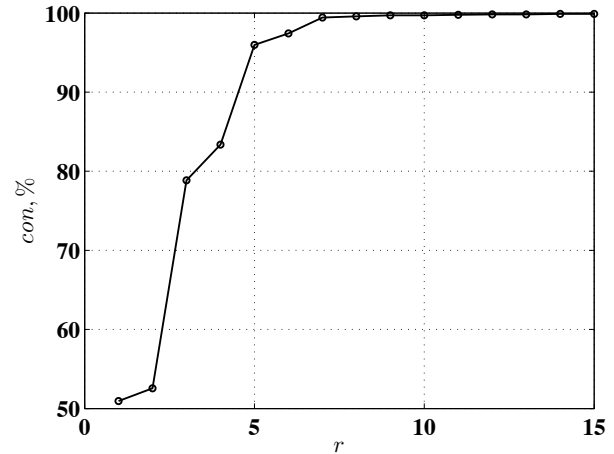

(c)

Fig. 9 Convergence indices con corresponding to the FRSs associated with the third mode in the (a) first, (b) second and (c) third half-scan periods. 


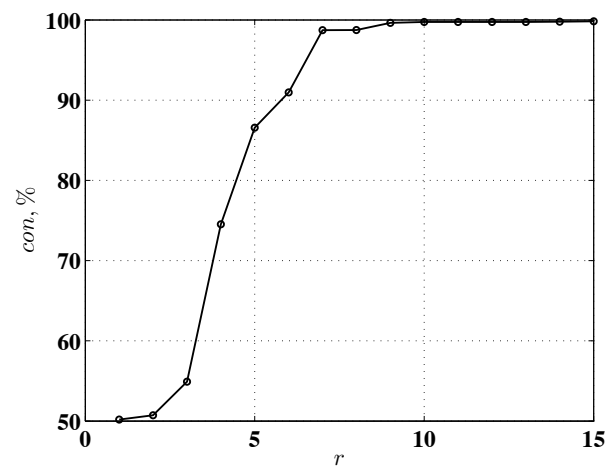

Fig. 10 Convergence index con corresponding to the FRS associated with the fourth mode in the first half-scan period.

FRDIs in Eq. (27) associated with the first through fourth modes are shown in Figs. 11(a) through (d), respectively, and the auxiliary FRDI in Eq. (28) is shown in Fig. 11(e). Note that numbers of FRSs included in the FRDIs associated with the first through fourth modes are 3, 3, 3 and 1, respectively. The damage can be clearly identified near neighborhoods with consistently high values of the FRDIs and that with high values of the auxiliary FRDI. 


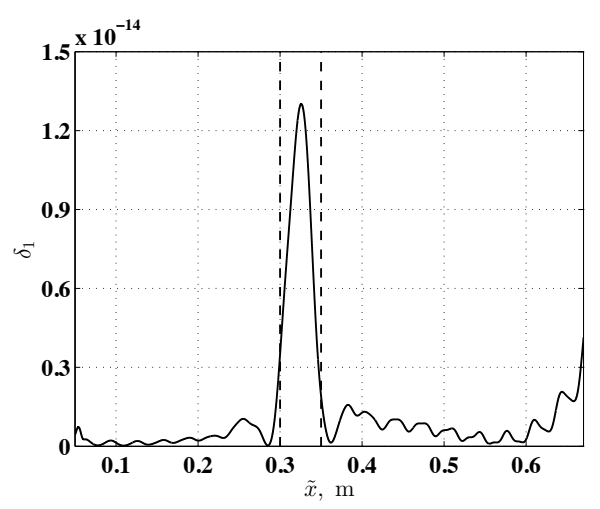

(a)

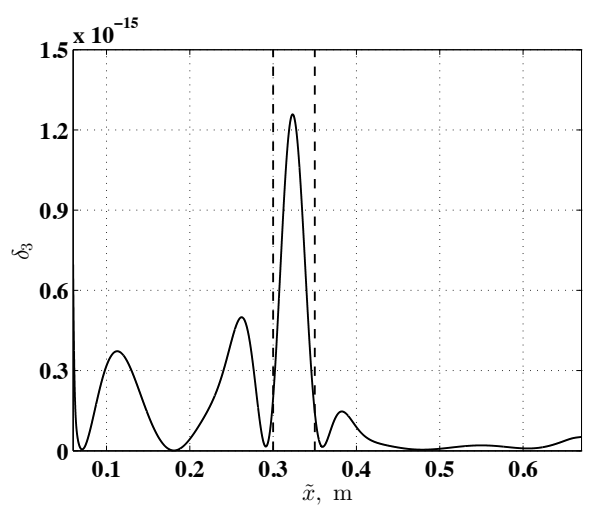

(c)

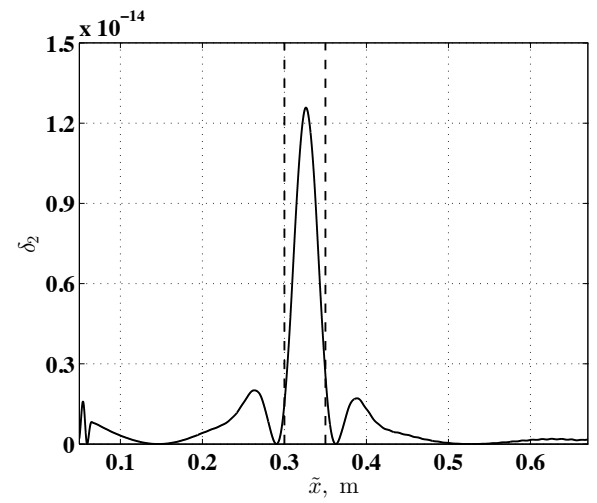

(b)

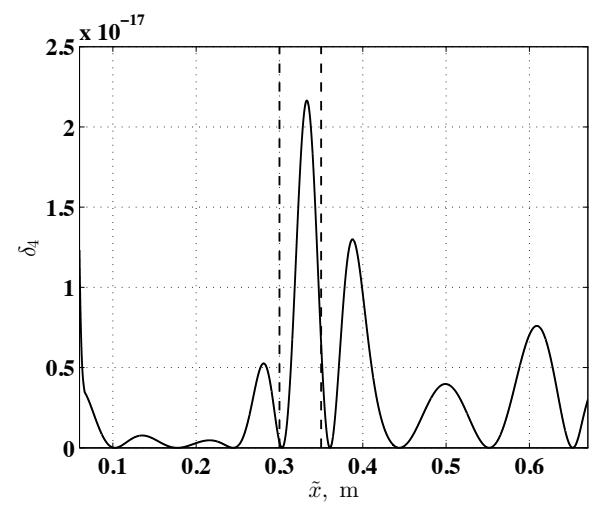

(d)

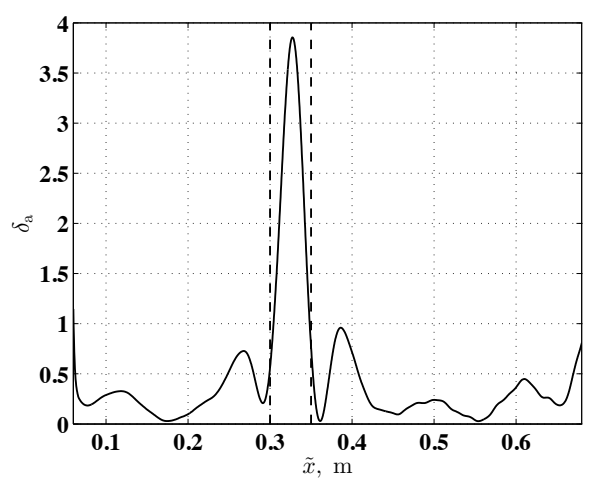

(e)

Fig. 11 FRDIs associated with the (a) first, (b) second, (c) third and (d) fourth modes of the damaged beam structure, and (e) the auxiliary FRDI associated with the four modes. Locations of damage ends are indicated by two vertical dashed lines. 


\section{Experimental Investigation}

\subsection{CSLDV system}

A CSLDV system shown in Fig. 12 is developed in this work. It consists of a Cambridge $6240 \mathrm{H}$ scanner, a Polytec OFV-353 single-point laser vibrometer and a dSPACE MicroLabBox controller board that controls a pair of orthogonal scan mirrors of the scanner termed as X and Y mirrors; the control software ControlDesk Next Generation is used to design and implement a control scheme for the system. The mirrors are connected to two independent stepper motors in the scanner. Input signals to each stepper motor directly control rotation angles of the mirrors, and different scan patterns of the laser spot can be created. In the control scheme, triangular and constant input signals are given to the $\mathrm{X}$ and $\mathrm{Y}$ mirrors here, respectively, and the laser spot is continuously swept along the length of a beam structure. It can be assumed that the resultant velocity of the laser spot on the structure is constant along a scan line, when the system is sufficiently far away from the structure and the rotation angle amplitude is sufficiently small. A triggering sub-scheme is embedded in the control scheme using a toolbox of the control software called Signal Editor. Starts of continuous scanning and measurement of the CSLDV system are triggered, when it registers a voltage with an absolute value higher than a prescribed threshold via the laser vibrometer. 


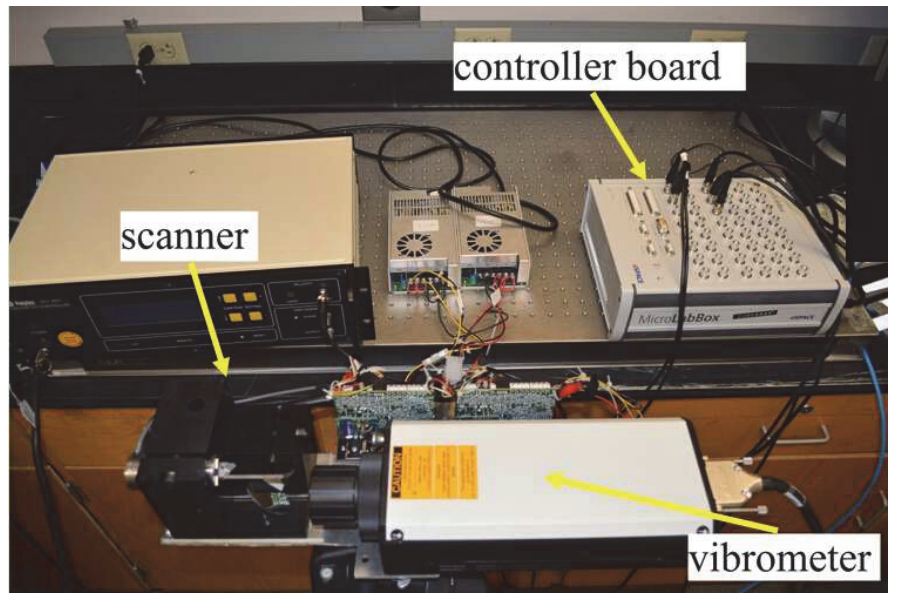

Fig. 12 A CSLDV system developed in this work.

An experiment was set up to obtain FRSs of a damaged aluminum cantilever beam structure using the CSLDV system for damage identification. A schematic diagram that shows the experimental setup and dimensions of the structure is shown in Fig. 13(a). The undamaged portion of the structure had a thickness of $6.35 \mathrm{~mm}$, and there was a region of machined thickness reduction of $1.27 \mathrm{~mm}$ on one side of the structure along its length, as shown in Figs. 13(a) and (b). The damage was selected in this form in order to show that its region can be accurately identified by the proposed methodology. The thickness reduction is about $20 \%$ of the thickness of the undamaged portion; its location and length are shown in Fig. 13(a). A bench vice was used to clamp the left end of the structure to simulate a fixed boundary. A straight scan line was assigned on the intact side of the structure along its length as shown in Fig. 13(a) and (c). A strip of retroreflective tape was attached on the intact side to enhance laser reflection that directly determined signal-to-noise ratios of measurement by the CSLDV system. The scan line 
was non-dimensionalized to range from $0 \%$ to $100 \%$, where $0 \%$ and $100 \%$ represented left and right ends of the scan line, respectively. The damage was located between $45.71 \%$ and $51.43 \%$ on the scan line. The experimental setup is shown in Figs. 13(a), (c) and (d), where the CSLDV system was used to measure free response of the structure along the scan line. In this work, the sampling frequency of the system was $250,000 \mathrm{~Hz}$, and the threshold for triggering was $0.1 \mathrm{~V}$. The laser spot of the system stayed at $80 \%$ on the scan line before its starts of continuous scanning and measurement were triggered, and continuous scanning started by sweeping the laser spot towards $100 \%$ on the scan line. 


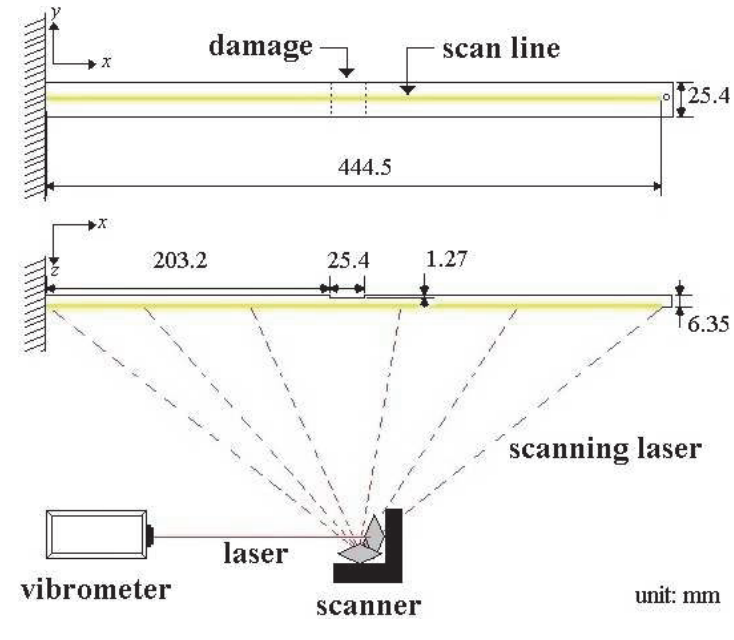

(a)

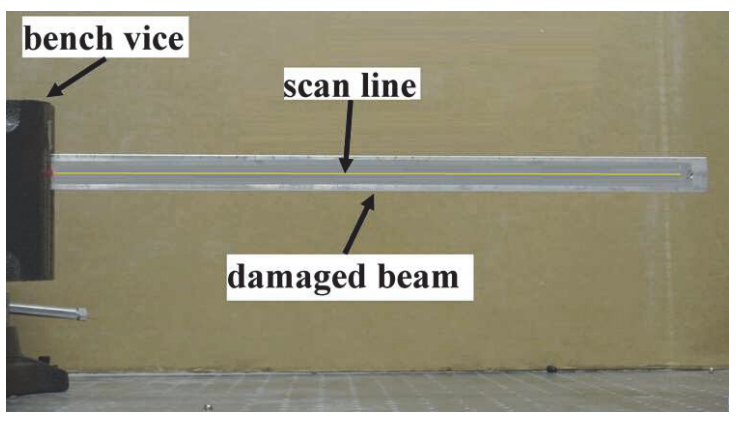

(c)

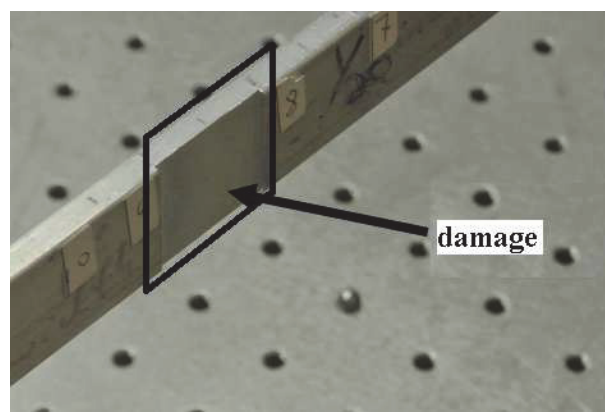

(b)

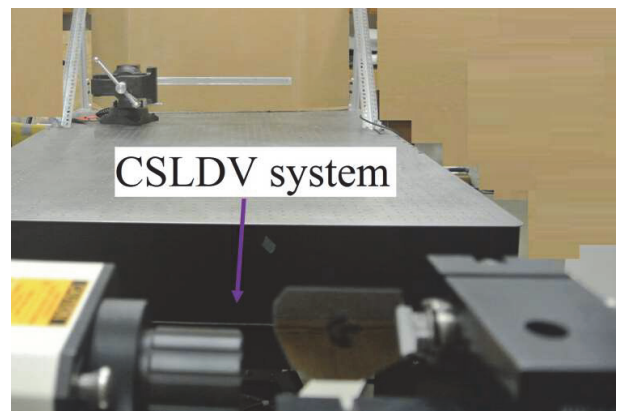

(d)

Fig. 13 (a) Schematic diagram of the test setup and dimensions of a damaged aluminum cantilever beam structure with a region of machined thickness reduction, (b) the region of machined thickness reduction, (c) the structure with its left end clamped by a bench vice and (d) the experimental setup for FRS measurement of the structure.

An impact test was first conducted on the beam structure in Fig. 13 to measure its first five natural frequencies; a PCB 086E80 pen-sized impact hammer and the single-point laser Doppler vibrometer in Fig. 12 were used 
to excite the structure at an impact point and measure its response at a measurement point, respectively. Both the impact and measurement points on the structure were arbitrarily selected as long as they did not coincide with nodal points of its first five modes. The first five natural frequencies of the structure were measured to be $22.07 \mathrm{~Hz}, 135.74 \mathrm{~Hz}, 393.36 \mathrm{~Hz}, 746.48$ $\mathrm{Hz}$ and $1261.07 \mathrm{~Hz}$.

Velocity response of the beam structure in Fig. 13 under two forms of excitation were measured by the CSLDV system with different scan frequencies. One is initial non-zero bending deformation of the structure along its length under a transverse concentrated force at its free end, with zero initial velocity, for damage identification using FRDIs associated with low modes of the structure; the other is an impact on the structure with zero initial conditions for damage identification using FRDIs associated with its high modes. The impact point was on the damaged side of the structure, and the distance between the impact point and fixed end of the structure was $80 \mathrm{~mm}$. FRSs of the structure associated with its first five modes were obtained from the demodulation method using velocity response measured by the CSLDV system with different scan frequencies of $0.1 \mathrm{~Hz}, 1.0 \mathrm{~Hz}$ and $10.0 \mathrm{~Hz}$. Effects of the scan frequency of the CSLDV system on obtained FRSs and damage identification results were investigated. As measurement noise exists in obtained FRSs and its adverse effects can be amplified in calculating associated curvature FRSs, a numerical smoothing technique was applied to alleviate the adverse effects before calculating the curvature FRSs, which is local regression using weighted linear least squares and a second-order polynomial model [33]. In the smoothing technique, weighted quadratic least squares 
are calculated at each measurement point within an interval that consists of a certain number of its neighboring points, which was $15 \%$ of the total number of measurement points in this investigation. It is the width of the interval that determines how good the technique is in smoothing an obtained FRS. One should be cautious about choosing the width, as the technique can smooth out effects of damage in a curvature FRS and compromise effectiveness of the proposed damage identification methodology if a chosen width is too large, though the method can still alleviate adverse effects of measurement noise on the curvature FRS. Unfortunately, a general guideline for choosing an optimal width of the interval for damage identification does not exist, as existence of damage and quality of an obtained FRS are usually unknown in practice. Hence, one should test different widths of the interval from small to large ones and compare resulting FRDIs associated with different modes. If signal-to-noise ratios of FRS measurements are adequately high, it would be adverse effects of measurement noise on curvature FRSs that are first smoothed out by the technique, and effects of damage would be preserved in the curvature FRSs and observed in resulting FRDIs. In this case, damage can be clearly identified in neighborhoods with consistently

high values of the FRDIs in Eq. (27) and in those with high values of the auxiliary FRDI in Eq. (28).

\subsection{FRS measurement and damage identification results}

Velocity response of the beam structure under the initial non-zero bending deformation was measured by the CSLDV system with a scan frequency of $0.1 \mathrm{~Hz}$. The measured response and X-mirror feedback signal are shown in Figs. 14(a) and (b), respectively, and a spectrogram of the response is shown 
in Fig. 14(c). It can be seen from Fig. 14(c) that STFTs at the second through fifth modes of the structure drastically decayed in less than one second after the scan started. Since the length of a half-scan period in this scan is five seconds, FRSs associated with the second through fifth modes could not be obtained by the system with the scan frequency, while those associated with the first mode could be. FRSs associated with the first mode in the first three half-scan periods are shown in Fig. 15. Similar to those in the numerical investigation, amplitudes of the FRSs decreased from one half-scan period to the next. 


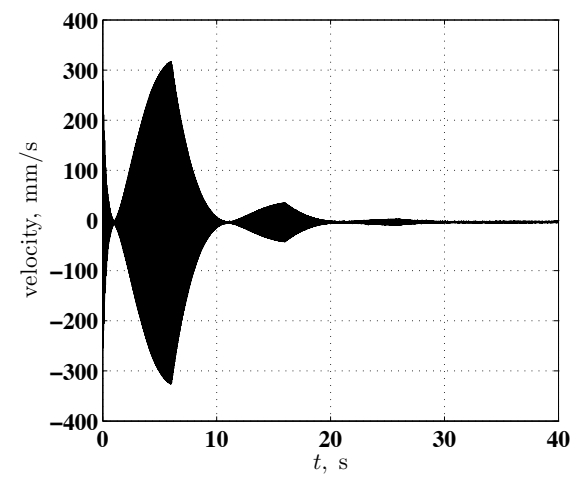

(a)

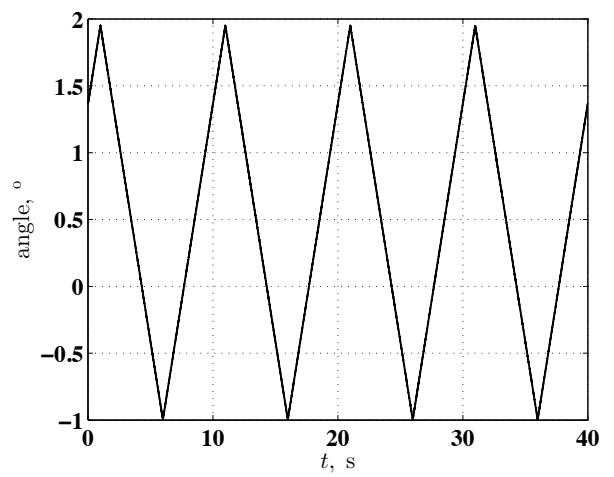

(b)

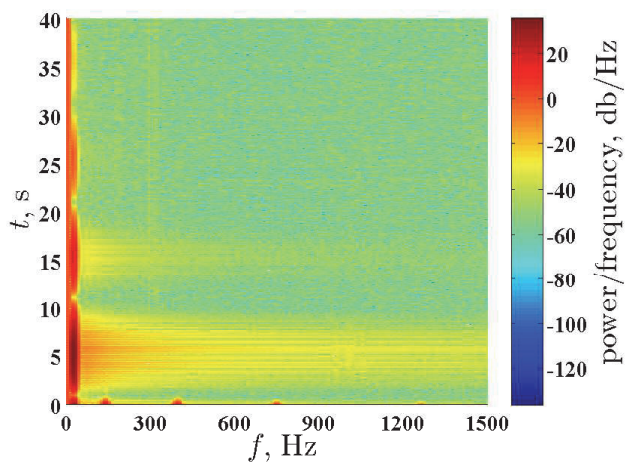

(c)

Fig. 14 (a) Velocity response of the beam structure under the initial non-zero bending deformation measured by the CSLDV system with a scan frequency of $0.1 \mathrm{~Hz}$, (b) the X-mirror feedback signal with a triangular input signal and (c) a spectrogram of the response in (a). 


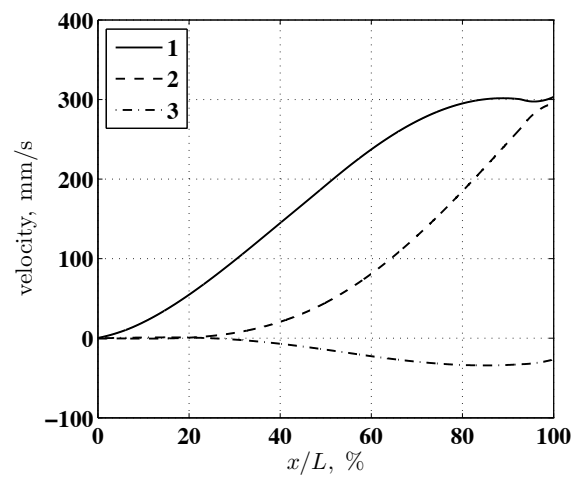

Fig. 15 Obtained FRSs associated with the first mode in the first three half-scan periods of the CSLDV system with a scan frequency of $0.1 \mathrm{~Hz}$.

Velocity response of the beam structure under the impact was measured by the CSLDV system with a scan frequency of $1.0 \mathrm{~Hz}$. The measured response and X-mirror feedback signal are shown in Figs. 16(a) and (b), respectively, and a spectrogram of the response is shown in Fig. 16(c). It can be seen from Fig. 16(c) that STFTs at the second through fifth natural frequencies of the beam drastically decayed in the first three, two, one and half seconds of the scan, respectively. FRSs associated with the fifth natural frequency of the structure could not be obtained, since the length of a halfscan period in this case is $0.5 \mathrm{~s}$ and the first half scan ended at $t=0.6$ s. FRSs associated with the first through fourth modes in the first three half-scan periods are shown in Fig. 15(a) through (d), respectively. 


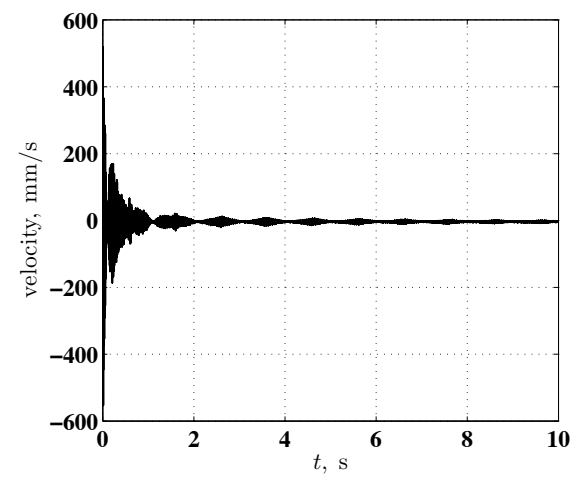

(a)

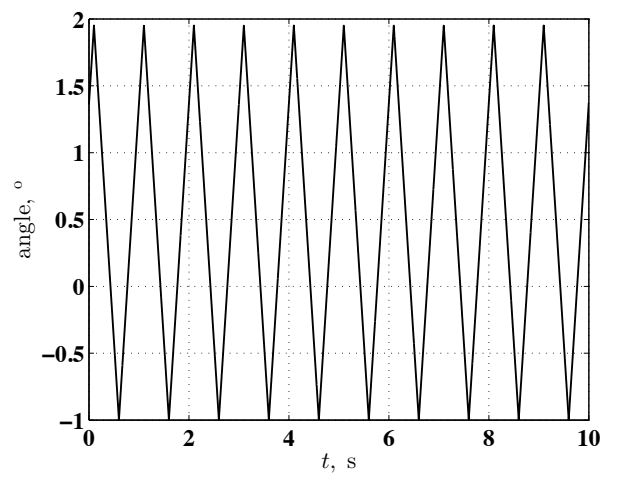

(b)

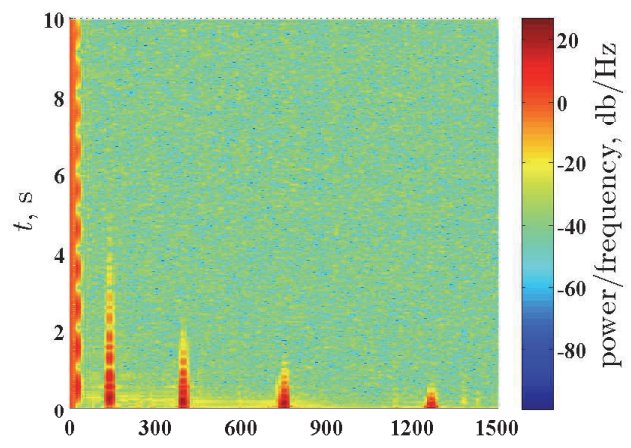

(c)

Fig. 16 (a) Velocity response of the beam structure under the impact measured by the CSLDV system with a scan frequency of $1.0 \mathrm{~Hz}$, (b) the X-mirror feedback signal with a triangular input signal and (c) a spectrogram of the response in (a). 


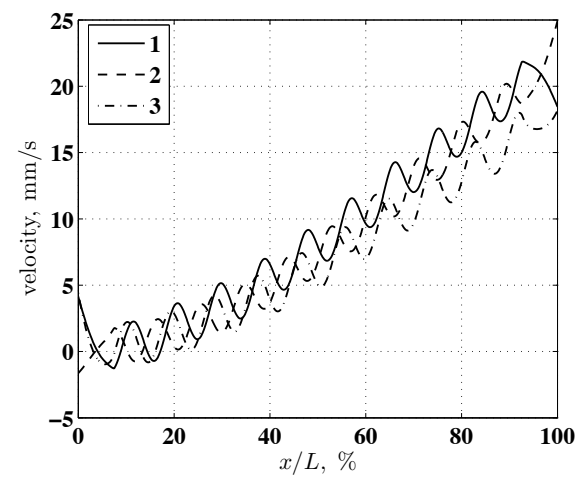

(a)

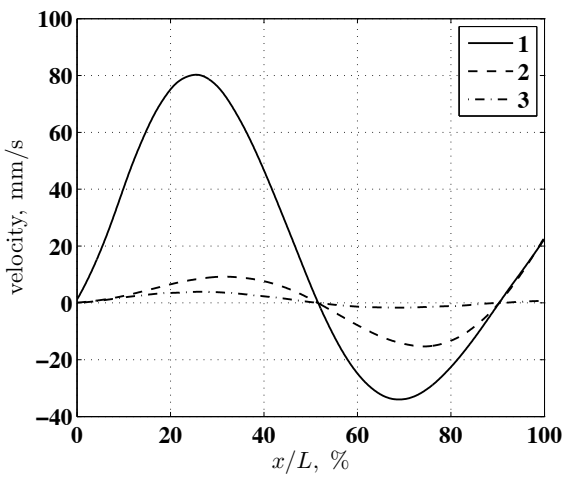

(c)

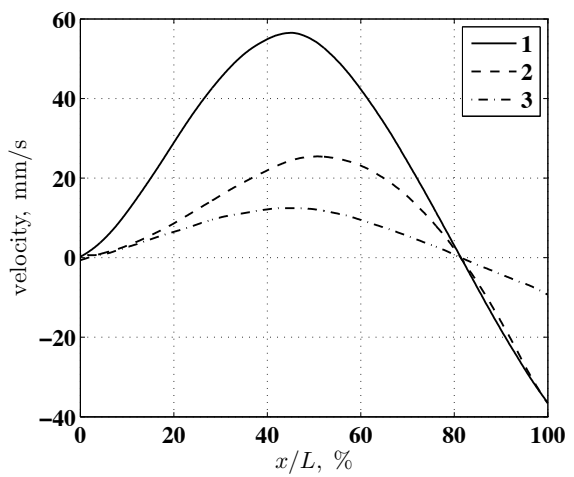

(b)

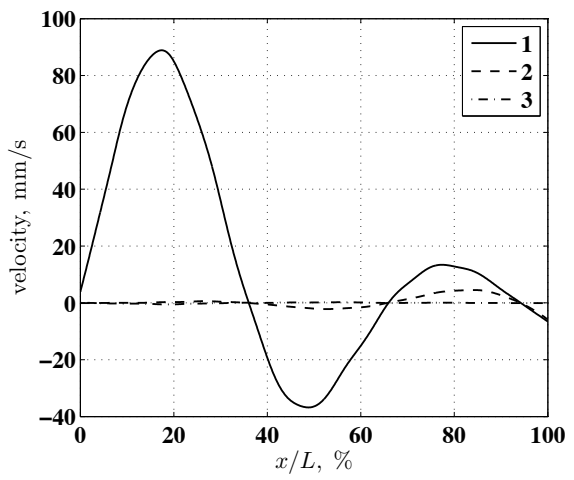

(d)

Fig. 17 Obtained FRSs associated with the (a) first, (b) second, (c) third and (d) fourth modes in the first three half-scan periods of the CSLDV system with a scan frequency of $1.0 \mathrm{~Hz}$.

Velocity response of the beam structure under the impact was then measured by the CSLDV system with a scan frequency of $10.0 \mathrm{~Hz}$. The measured response and X-mirror feedback signal are shown in Figs. 18(a) and (b), respectively, and a spectrogram of the response are shown in Fig. 18(c). It can be seen from Fig. 18(c) that STFTs at the second through fifth natural fre- 
quencies of the structure drastically decayed in the first three, two, one and half seconds of the scan, respectively. FRSs associated with the first through fourth modes in the first three half-scan periods are shown in Figs. 19(a) through (e), respectively. A critical observation can be made by comparing Figs. 16(a) and 18(a) that the measured response by the CSLDV system with a scan frequency of $1.0 \mathrm{~Hz}$ had a higher signal-to-noise ratio than that by the system with a scan frequency of $10.0 \mathrm{~Hz}$, as the former seemed to be dominated by measurement noise after $t=9 \mathrm{~s}$ and the latter after $t=1.5 \mathrm{~s}$. Since the structure was under the same impact in the measurement, the theory that speckle noise in measurement by a CSLDV system, which directly results in a lower signal-to-noise ratio in its measurement and lower-quality MSs, increases with its scan frequency [34, 35] was validated. 


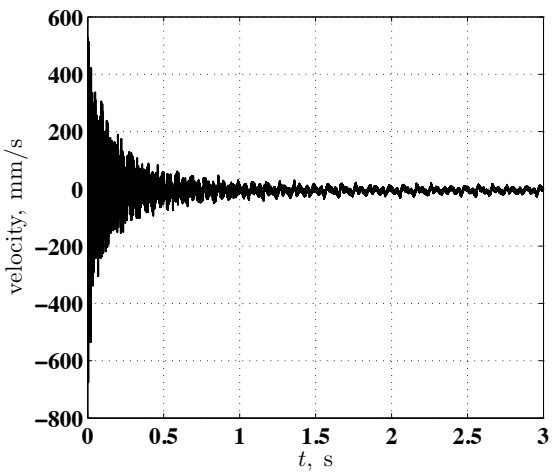

(a)

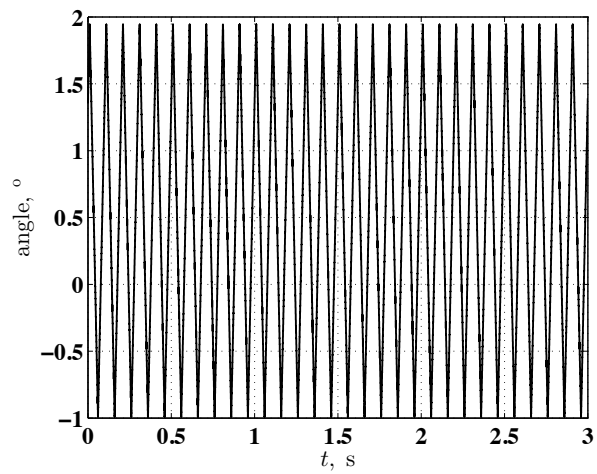

(b)

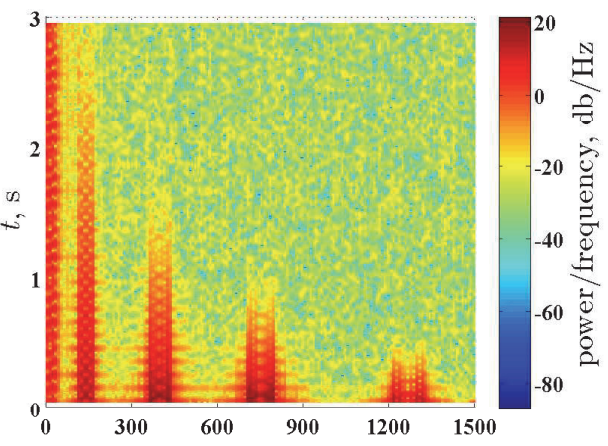

(c)

Fig. 18 (a) Velocity response of the beam structure under the impact measured by the CSLDV system with a scan frequency of $10.0 \mathrm{~Hz}$, (b) the X-mirror feedback signal with a triangular input signal and (c) a sprectrogram of the response in (a). 


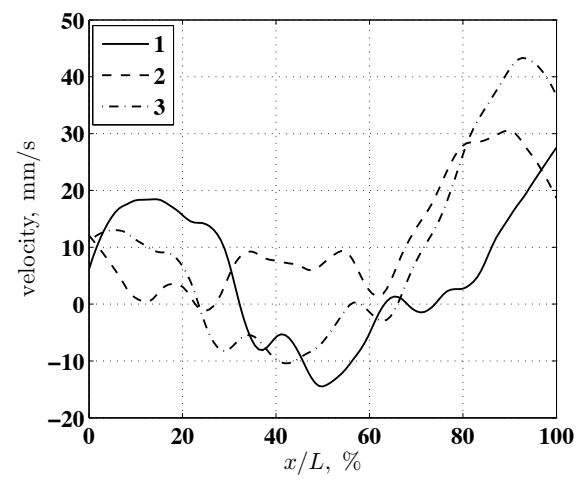

(a)

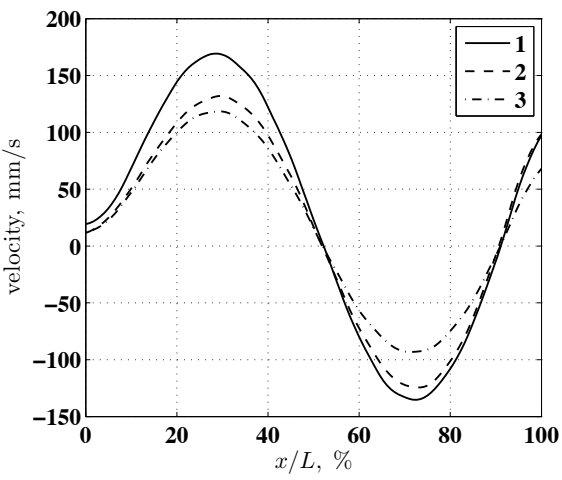

(c)

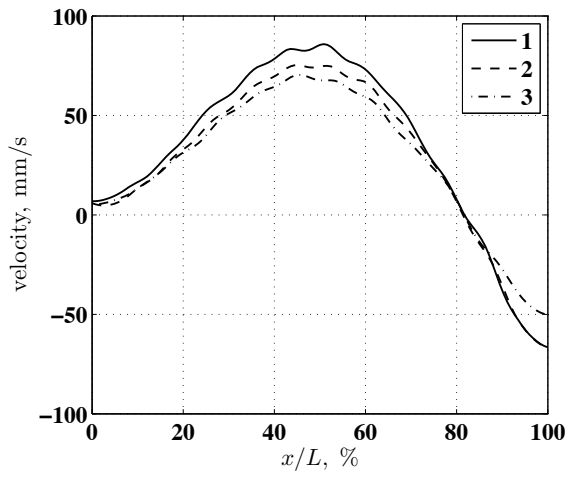

(b)

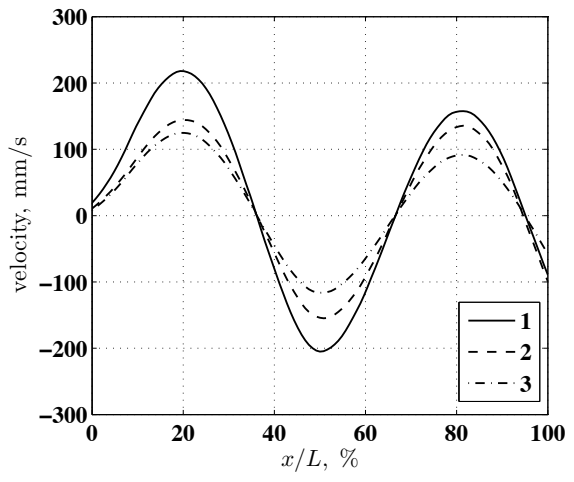

(d)

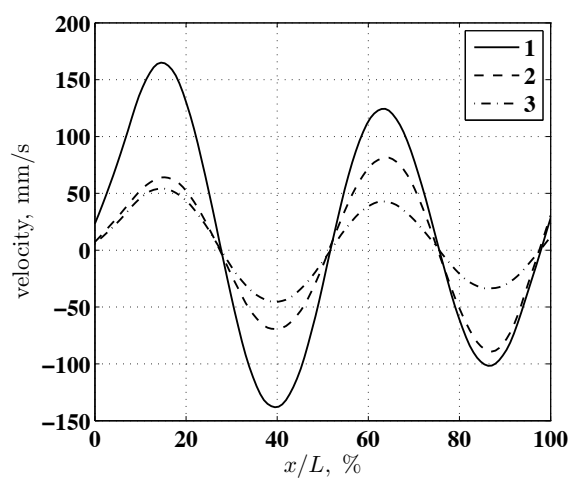

(e)

Fig. 19 Obtained FRSs associated with the (a) first, (b) second, (c) third, (d) fourth and (e) fifth modes in the first three half-scan periods of the CSLDV system with a scan frequency of $10.0 \mathrm{~Hz}$. 
Effects of the scan frequency on qualities of FRSs of the beam structure can be seen by comparing FRSs of the structure associated with its first through fourth modes obtained by use of the CSLDV system with different scan frequencies, as shown in Figs. 15, 17 and 19. For the FRSs associated with the first mode, their qualities were the best when a low scan frequency of $0.1 \mathrm{~Hz}$ was used, and their qualities became worse when the CSLDV system had a higher scan frequency. Similar observations can be made for the FRSs associated with the second and third modes, since their qualities when the scan frequency was $1.0 \mathrm{~Hz}$ were better than those when the scan frequency was $10.0 \mathrm{~Hz}$. One can conclude that effects of the scan frequency on qualities of FRSs are similar to those on qualities of MSs: increasing the scan frequency of a CSLDV system can lower qualities of obtained FRSs. Hence, it is recommended that a low scan frequency be used to measure response of a structure for obtaining its FRSs at a low natural frequency, as long as the scan period corresponding to the scan frequency of a CSLDV system is large enough for it to measure the response of at least one half-scan period at the natural frequency. FRDIs in Eq. (27) associated with the first five modes of the structure were calculated and shown in Fig. 20(a) through (e) using the FRSs obtained by use of the CSLDV system with different scan frequencies in different numbers of half-scan periods, and the associated auxiliary FRDI in Eq. (28) was calculated and shown in Fig. 20(f). The damage can be clearly identified near neighborhoods with consistently high values of the FRDIs and that with high values of the auxiliary FRDI. Scan frequencies and numbers of half-scan periods associated with different modes of the structure for calculating the FRDIs are listed in Table 3. 


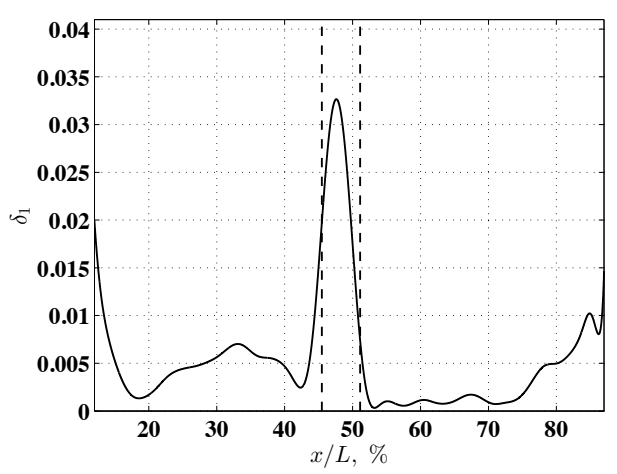

(a)

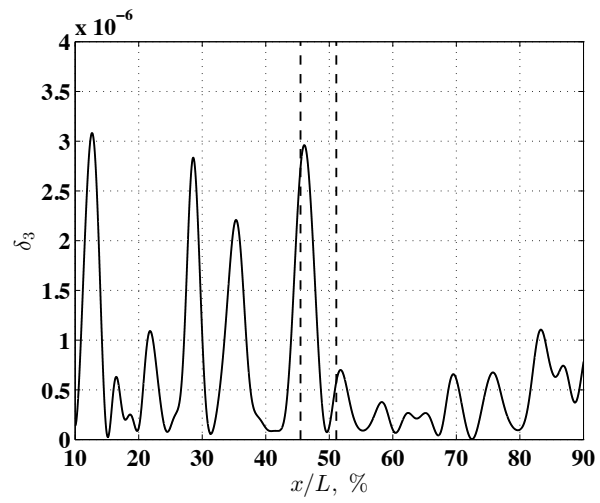

(c)

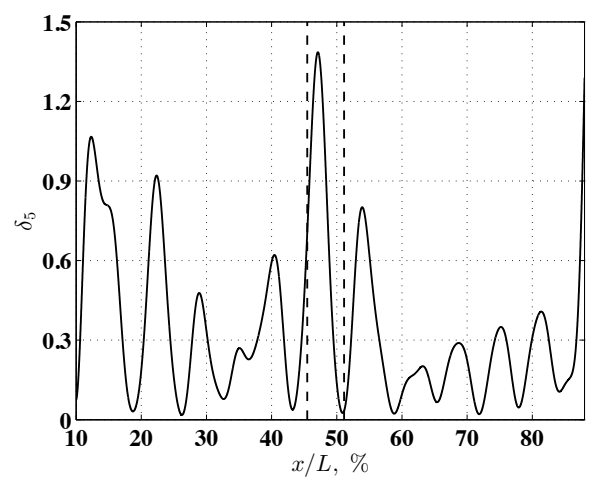

(e)

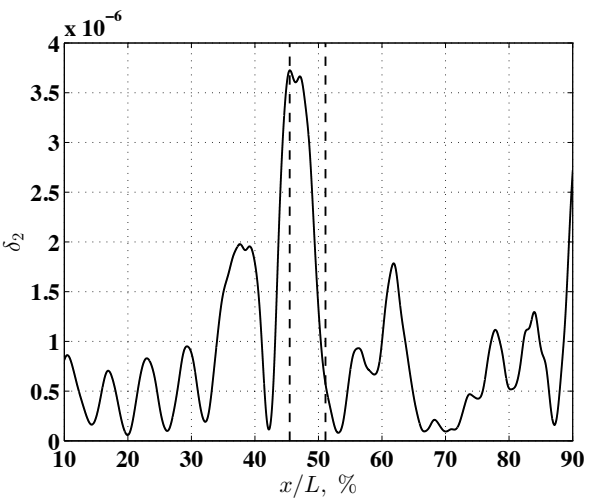

(b)

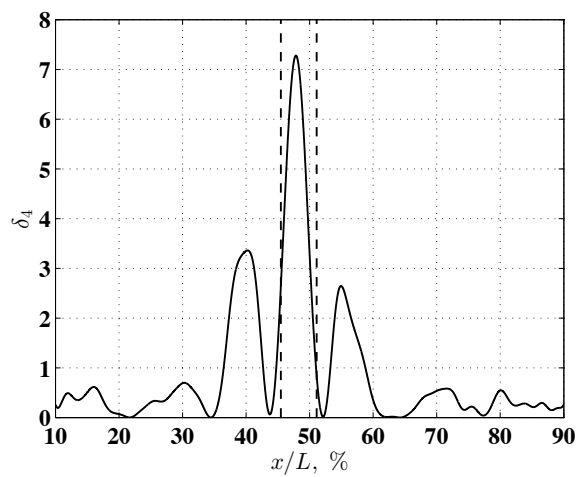

(d)

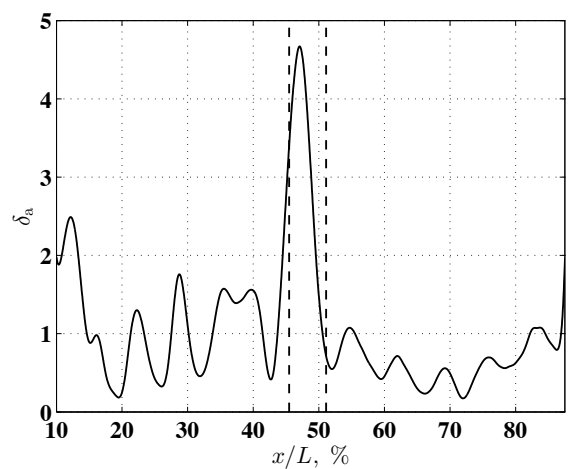

(f)

Fig. 20 FRDIs associated with the FRSs associated with the (a) first, (b) second, (c) third, (d) fourth and (e) fifth modes, and (f) the auxiliary FRDI associated with the five modes. Locations of damagqends are indicated by two vertical dashed lines. 
Tab. 3 Scan frequencies and numbers of half-scan periods for calculating FRDIs associated with the first five modes of the beam structure.

\begin{tabular}{|c|c|c|c|c|c|}
\hline Mode & 1 & 2 & 3 & 4 & 5 \\
\hline Scan frequency (Hz) & 0.1 & 1.0 & 1.0 & 10.0 & 10.0 \\
\hline Number of half-scan periods & 5 & 4 & 3 & 4 & 3 \\
\hline
\end{tabular}

\section{Conclusion}

A new type of vibration shapes called a FRS is introduced in this work. A FRS can be obtained by use of a CSLDV system, and it can be obtained from the demodulation method using free response of a structure. An analytical expression of a FRS is derived for a beam structure with damping that can be modeled by the Kelvin-Voigt viscoelastic model. FRSs from the analytical expression compare well with those from a FE model. A FRDI that uses differences between curvatures of FRSs associated with a mode and those from polynomial fits is proposed, and damage regions can be accurately identified near neighboorhoods with consistently high values of FRDIs associated with different modes; an auxiliary FRDI can assist identification of the neighborhoods. A polynomial fits a FRS of a damaged beam structure whose order can be properly determined using a convergence index, and a FRS from the polynomial fit can be considered to be that of an undamaged beam structure, if the undamaged structure is geometrically smooth and made of materials that have no stiffness and mass discontinuities. It was numerically and experimentally shown that amplitudes of FRSs decrease from one half-scan 
period to the next, and spectrograms of response measured by a CSLDV system can be used to determine instants before which non-zero FRSs can be obtained. Effects of the scan frequency of a CSLDV system were experimentally investigated, and it was observed that a lower scan frequency could yield higher-quality FRSs and a higher scan frequency could yield lowerquality FRSs; it is recommended that a low scan frequency be used for FRSs at a low natural frequency. The proposed methodology was numerically and experimentally applied to damaged beam structures with machined thickness reductions along their lengths. Damage regions were successfully identified near neighborhoods with consistently high values of FRDIs associated with different modes and that with high values of the auxiliary FRDI.

\section{Acknowledgement}

The authors are grateful for the financial support from the National Science Foundation under Grant Numbers CMMI-1229532 and CMMI-1335024 and the College of Engineering and Information Technology at the University of Maryland, Baltimore County through a Strategic Plan Implementation Grant.

\section{References}

[1] W. Fan, P. Qiao, Vibration-based damage identification methods: a review and comparative study, Structural Health Monitoring 10 (1) (2011) $83-111$.

[2] D. J. Ewins, Modal Testing: Theory, Practice and Application, 2nd Edition, Research Studies Press, Hertfordshire, UK, 2000. 
[3] C. N. Wong, W. D. Zhu, G. Y. Xu, On an iterative general-order perturbation method for multiple structural damage detection, Journal of Sound and Vibration 273 (1) (2004) 363-386.

[4] G. Y. Xu, W. D. Zhu, B. H. Emory, Experimental and numerical investigation of structural damage detection using changes in natural frequencies, Journal of Vibration and Acoustics 129 (6) (2007) 686-700.

[5] K. He, W. D. Zhu, Detection of damage and loosening of bolted connections in structures using changes in natural frequencies, ASNT Materials Evaluation 68 (6) (2010) 721-732.

[6] K. He, W. D. Zhu, Vibration-based structural damage detection method and its applications to engineering structures, International Journal of Smart and Nano Materials 2 (3) (2011) 194-218.

[7] W. D. Zhu, K. He, Detection of damage in space frame structures with l-shaped beams and bolted joints using changes in natural frequencies, Journal of Vibration and Acoustics 135 (5) (2013) 051001.

[8] K. He, W. D. Zhu, Detecting loosening of bolted connections in a pipeline using changes in natural frequencies, Journal of Vibration and Acoustics 136 (3) (2014) 034503.

[9] C. Surace, R. Archibald, R. Saxena, On the use of the polynomial annihilation edge detection for locating cracks in beam-like structures, Computers \& Structures 114 (2013) 72-83.

[10] A. K. Pandey, M. Biswas, M. M. Samman, Damage detection from 
changes in curvature mode shapes, Journal of Sound and Vibration 145 (2) (1991) 321-332.

[11] C. P. Ratcliffe, W. J. Bagaria, Vibration technique for locating delamination in a composite beam, AIAA journal 36 (6) (1998) 1074-1077.

[12] C. P. Ratcliffe, A frequency and curvature based experimental method for locating damage in structures, Journal of Vibration and Acoustics 122 (3) (2000) 324-329.

[13] M.-K. Yoon, D. Heider, J. W. Gillespie Jr, C. P. Ratcliffe, R. M. Crane, Local damage detection with the global fitting method using mode shape data in notched beams, Journal of Nondestructive Evaluation 28 (2) (2009) 63-74.

[14] M.-K. Yoon, D. Heider, J. W. Gillespie Jr, C. P. Ratcliffe, R. M. Crane, Local damage detection with the global fitting method using operating deflection shape data, Journal of Nondestructive Evaluation 29 (1) (2010) 25-37.

[15] M.-K. Yoon, D. Heider, J. W. Gillespie Jr, C. P. Ratcliffe, R. M. Crane, Local damage detection using the two-dimensional gapped smoothing method, Journal of Sound and Vibration 279 (1) (2005) 119-139.

[16] Y. F. Xu, W. D. Zhu, J. Liu, Y. M. Shao, Identification of embedded horizontal cracks in beams using measured mode shapes, Journal of Sound and Vibration 333 (23) (2014) 6273-6294.

[17] S. Rothberg, J. Baker, N. A. Halliwell, Laser vibrometry: pseudovibrations, Journal of Sound and Vibration 135 (3) (1989) 516-522. 
[18] P. Sriram, S. Hanagud, J. Craig, N. Komerath, Scanning laser doppler technique for velocity profile sensing on a moving surface, Applied Optics 29 (16) (1990) 2409-2417.

[19] P. Sriram, S. Hanagud, J. Craig, Mode shape measurement using a scanning laser doppler vibrometer 7 (3) (1992) 169-178.

[20] A. Stanbridge, D. Ewins, Using a continuously-scanning laser doppler vibrometer for modal testing, in: Proceedings of the International Modal Analysis Conference, 1996, pp. 816-822.

[21] A. Stanbridge, D. Ewins, Modal testing using a scanning laser doppler vibrometer, Mechanical Systems and Signal Processing 13 (2) (1999) $255-270$.

[22] A. Stanbridge, D. Ewins, A. Khan, Modal testing using impact excitation and a scanning ldv, Shock and Vibration 7 (2) (2000) 91-100.

[23] D. Di Maio, D. Ewins, Continuous scan, a method for performing modal testing using meaningful measurement parameters: Part i, Mechanical Systems and Signal Processing 25 (8) (2011) 3027-3042.

[24] M. S. Allen, M. W. Sracic, A new method for processing impact excited continuous-scan laser doppler vibrometer measurements, Mechanical Systems and Signal Processing 24 (3) (2010) 721-735.

[25] S. Yang, M. S. Allen, Lifting approach to simplify output-only continuous-scan laser vibrometry, Mechanical Systems and Signal Processing 45 (2) (2014) 267-282. 
[26] A. Khan, A. B. Stanbridge, D. J. Ewins, Detecting damage in vibrating structures with a scanning ldv, Optics and Lasers in Engineering 32 (6) (1999) 583-592.

[27] D.-M. Chen, Y. F. Xu, W. D. Zhu, Damage identification of beams using a continuous scanning laser doppler vibrometer system, Journal of Vibration and Acoustics.

[28] L. Meirovitch, Principles and techniques of vibrations, Prentice Hall New Jersey, 1997.

[29] T. K. Caughey, M. E. J. Okelly, Classical normal modes in damped linear dynamic systems, Journal of Applied Mechanics 32 (3) (1965) $583-588$.

[30] S. S. Rao, Mechanical Vibrations, 4th Edition, Pearson Prentice Hall, 2004.

[31] F. Hlawatsch, F. Auger, Time-frequency Analysis, John Wiley \& Sons, 2013.

[32] I. Cox, M. Gaudard, Discovering Partial Least Squares with JMP, SAS Institute, 2013.

[33] M. U. Guide, The mathworks, Inc., Natick, MA 5 (1998) 333.

[34] M. W. Sracic, M. S. Allen, Experimental investigation of the effect of speckle noise on continuous scan laser doppler vibrometer measurements, in: Proceedings of the International Modal Analysis Conference, 2009, pp. $9-12$. 
[35] S. Yang, Modal identification of linear time periodic systems with applications to continuous-scan laser doppler vibrometry, Ph.D. thesis, Univeristy of Wisconsin-Madison (2013). 